\title{
Traditional use of medicinal plants in south-central Zimbabwe: review and perspectives
}

Alfred Maroyi

\begin{abstract}
Background: Traditional medicine has remained as the most affordable and easily accessible source of treatment in the primary healthcare system of resource poor communities in Zimbabwe. The local people have a long history of traditional plant usage for medicinal purposes. Despite the increasing acceptance of traditional medicine in Zimbabwe, this rich indigenous knowledge is not adequately documented. Documentation of plants used as traditional medicines is needed so that the knowledge can be preserved and the utilized plants conserved and used sustainably. The primary objective of this paper is to summarize information on traditional uses of medicinal plants in south-central Zimbabwe, identifying research gaps and suggesting perspectives for future research.

Methods: This study is based on a review of the literature published in scientific journals, books, reports from national, regional and international organizations, theses, conference papers and other grey materials.

Results: A total of 93 medicinal plant species representing 41 families and 77 genera are used in south-central Zimbabwe. These plant species are used to treat 18 diseases and disorder categories, with the highest number of species used for gastro-intestinal disorders, followed by sexually transmitted infections, cold, cough and sore throat and gynaecological problems. Shrubs and trees (38\% each) were the primary sources of medicinal plants, followed by herbs (21\%) and climbers (3\%). The therapeutic claims made on medicinal plants documented in south-central Zimbabwe are well supported by literature, with $82.8 \%$ of the plant species having similar applications in other regions of Zimbabwe as well as other parts of the world and $89.2 \%$ having documented biological and pharmacological properties.
\end{abstract}

Conclusion: This study illustrates the importance of traditional medicines in the treatment and management of human diseases and ailments in south-central Zimbabwe. Traditional medicines still play an important role in meeting basic health care of local communities in Zimbabwe.

Keywords: Conservation, Medicinal plants, South-central Zimbabwe, Traditional knowledge

\section{Background}

Out of more than 5000 plant species growing in Zimbabwe, about 10 percent of these have medicinal properties and are used as traditional medicines [1]. Traditional medicine has remained as the most affordable and easily accessible source of treatment in the primary healthcare system of resource poor communities in Zimbabwe. About $80 \%$ of the population in developing countries use traditional medicines because they cannot afford the high cost of western pharmaceuticals and health care, and because traditional medicines are

Correspondence: alfred.maroyi@gmail.com

Department of Biodiversity, School of Molecular and Life Sciences, University of Limpopo, Mankweng, South Africa more acceptable from a cultural and spiritual perspective [2]. Research by Hostettmann et al. [3] showed that the knowledge on the use of medicinal plants is enormous but if this traditional knowledge is not rapidly researched and recorded, indications are that it will be lost with succeeding generations. Despite the increasing acceptance of traditional medicine in Zimbabwe $[1,4,5]$, this rich indigenous knowledge on traditional remedies is not adequately documented. Documentation of plants used as traditional medicines in Zimbabwe is urgent so that the knowledge can be preserved, the utilized plants are conserved and used sustainably. The current investigation therefore, attempts to fill some of the gaps in indigenous knowledge related to the use of herbal medicines in

\section{() Biomed Central}

(c) 2013 Maroyi; licensee BioMed Central Ltd. This is an Open Access article distributed under the terms of the Creative Commons Attribution License (http://creativecommons.org/licenses/by/2.0), which permits unrestricted use, distribution, and reproduction in any medium, provided the original work is properly cited. 
south-central Zimbabwe (Figure 1) emphasizing their role in basic human health care. The majority of the people in south-central Zimbabwe belong to the Karanga dialectical group. Most of the Karanga people live in Mberengwa, Shurugwi and Zvishavane districts in the Midlands province; and Chivi and Zaka districts in the Masvingo province [7], which are the study sites of the current study. The Karanga people possess their own traditional knowledge on medicinal plants that contributes to a broader understanding of medicinal plants in Zimbabwe. This investigation is part of a larger study [8] aimed at documenting the ethnobotanical knowledge held by the Karanga people in south-central Zimbabwe. Therefore, this review was done to document traditional uses of medicinal plants in south-central Zimbabwe in order to provide comprehensive documentation, identify research gaps, and suggest perspectives for future research.

\section{Methods}

The traditional uses of medicinal plants in south-central Zimbabwe were collated. Available references or reports on the plant species were consulted from published scientific journals, books, reports from national, regional and international organizations, theses, conference papers and other grey materials. Literature was searched on international online databases such as ISI Web of Science, MEDLINE, Science Direct, Scopus and Google Scholar using specific search terms such as "medicinal plants", "traditional medicines", "Chivi or Mberengwa or Shurugwi or Zaka or Zvishavane districts", "Masvingo or Midlands provinces" and "Zimbabwe". References were also identified by searching the library collections of the National Herbarium and Botanic Gardens, Harare, Zimbabwe and University of Limpopo, South Africa. Data collected from the literature included: use(s), mode of preparation and administration of the species. Literature search was also done to document the biological and pharmacological activities of the documented plant species.

\section{Medicinal plant diversity}

This study recorded ninety three plant species as useful in traditionally managing various human diseases in south-central Zimbabwe (Table 1). Of these, 79 species are indigenous to Zimbabwe (84.9\%), while 14 species are exotic (15.1\%), either naturalized as weeds or cultivated in home gardens as ornamentals or food plants. Dicotyledons were dominant with 87 plant species (93.5\%), 5 monocotyledons (5.4\%) and 1 fern (1.1\%). These medicinal plants were distributed among 41 families and 77 genera. The majority of medicinal plants $(71,76.3 \%)$ used in southcentral Zimbabwe are from 19 families (Table 2). Plant families with the highest number of medicinal plants in south-central Zimbabwe were: Fabaceae sensu lato (11 species), followed by Anacardiaceae (9 species), Euphorbiaceae (7 species), Asteraceae, Ebenaceae and Tiliaceae

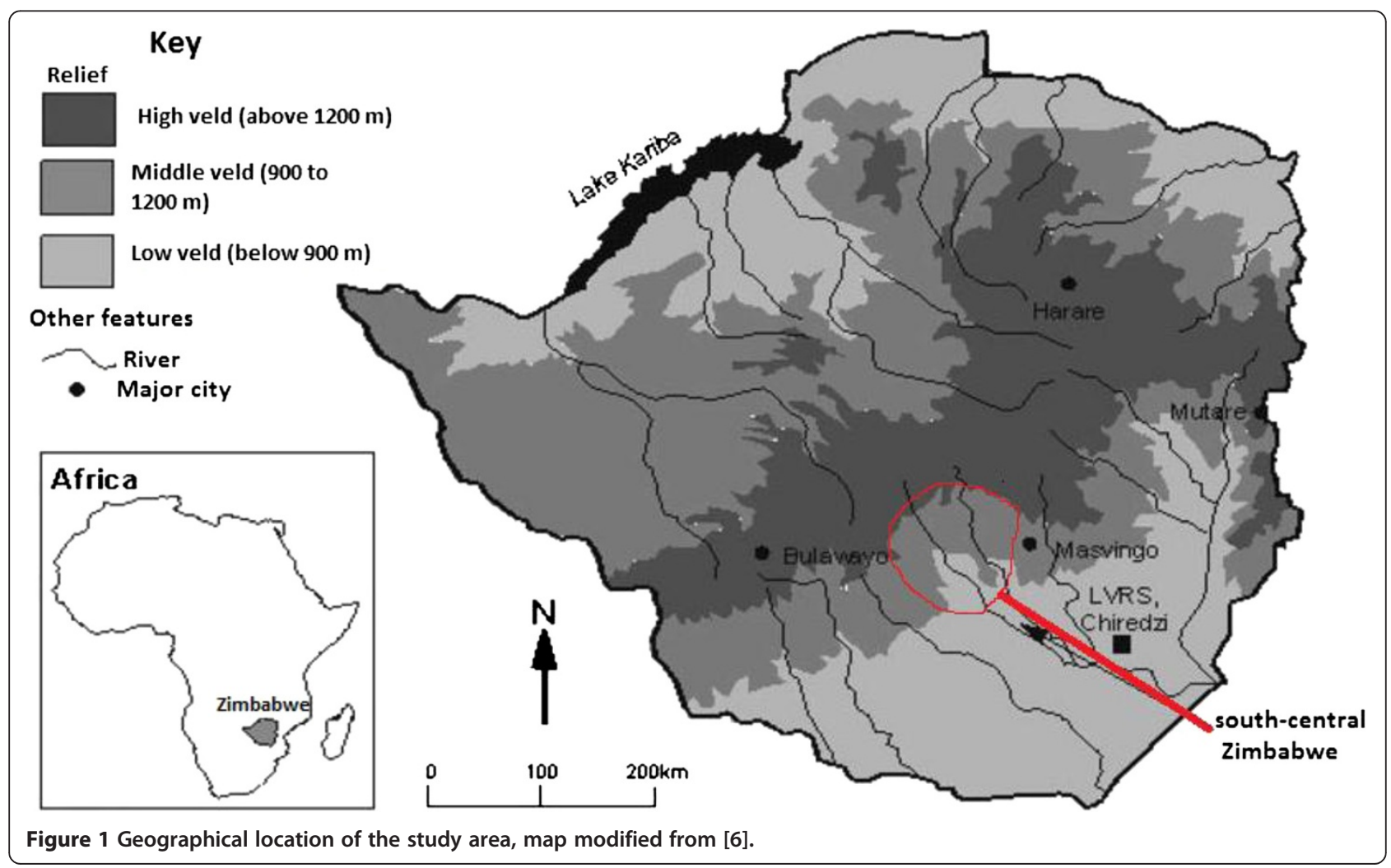


Table 1 Medicinal plants used in south-central Zimbabwe

\begin{tabular}{|c|c|c|c|c|c|}
\hline $\begin{array}{l}\text { Scientific name, } \\
\text { family }\end{array}$ & $\begin{array}{l}\text { Growth } \\
\text { habit }\end{array}$ & $\begin{array}{l}\text { Vernacular } \\
\text { name }\end{array}$ & Part(s) used and use(s) & $\begin{array}{l}\text { Similar uses in Zimbabwe } \\
\text { (other countries") } \\
\text { reported from literature }\end{array}$ & Reported biological/pharmacological activities \\
\hline \multicolumn{6}{|l|}{ Anacardiaceae } \\
\hline $\begin{array}{l}\text { Lannea discolor } \\
\text { (Sond.) Engl. }\end{array}$ & Tree & Mugan'acha & $\begin{array}{l}\text { Fibre: extract drunk to reduce the duration } \\
\text { of menstrual flow [9]. Roots: root extract } \\
\text { used as eye drops for sore eyes [8] }\end{array}$ & $\begin{array}{l}\text { Menorrhagia [1] and } \\
\text { infertility }{ }^{[1,10]}\end{array}$ & None found \\
\hline $\begin{array}{l}\text { Lannea edulis } \\
\text { (Sond.) Engl. }\end{array}$ & Shrub & Mutsambatsi & $\begin{array}{l}\text { Roots: extract drunk as bilharzia } \\
\text { (schistomiasis) and diarrhoea [9] and } \\
\text { gonorrhoea medicine [8] }\end{array}$ & $\begin{array}{l}\text { Gonorrhoea [1] and bilharzias }{ }^{\#,} \\
\text { diarrhoea }^{\#}[11]\end{array}$ & Antimicrobial activity; flavonoids and tannins [12] \\
\hline *Mangifera indica L. & Tree & Mumango & Bark: extract drunk as diarrhoea medicine [8] & Diarrhoea and dysentery [13] & $\begin{array}{l}\text { Antibacterial [13,14]; anti-inflammatory, } \\
\text { antifungal, antidiabetic, antioxidant, antiviral } \\
\text { and antiparasitic [14] properties }\end{array}$ \\
\hline Ozoroa insignis Del. & Shrub & Mubhedha & $\begin{array}{l}\text { Roots: extract drunk as diarrhoea and STIs } \\
\text { medicine [8] }\end{array}$ & $\begin{array}{l}\text { Diarrhoea and venereal } \\
\text { diseases [1] }\end{array}$ & $\begin{array}{l}\text { Antibacterial, anthelmintic }[15,16] \text {, antimicrobial } \\
{[16] \text { and cytotoxic [17] activities }}\end{array}$ \\
\hline Rhus longipes Engl. & Tree & Mufokosiana & $\begin{array}{l}\text { Roots: extract drunk as remedy for infertility } \\
\text { in women and to dilate birth canal [8] }\end{array}$ & $\begin{array}{l}\text { Infertility in women and } \\
\text { to dilate birth canal [1] }\end{array}$ & None found \\
\hline $\begin{array}{l}\text { Sclerocarya birrea } \\
\text { (A. Rich.) Hochst. }\end{array}$ & Tree & Mupfura & Roots: steam directed into sore eyes [8] & Sore eyes [1] & $\begin{array}{l}\text { Flavonoids, tannins and triterpenoids; } \\
\text { antidiarrhoeal, antidiabetic, anti-inflammatory, } \\
\text { antimicrobial and antioxidant [18] properties }\end{array}$ \\
\hline $\begin{array}{l}\text { Searsia dentata } \\
\text { (Thunb.) F.A. Barkley }\end{array}$ & Shrub & Mubikasadza & $\begin{array}{l}\text { Leaves: leaf sap taken as remedy for ulcers, } \\
\text { diarrhoea and stomach problems [9] }\end{array}$ & None found & Biflavonoids [19] \\
\hline $\begin{array}{l}\text { Searsia pyroides } \\
\text { (Burch.) Moffett }\end{array}$ & Shrub & Mufokosiana & Roots: extract drunk as cough medicine [8] & None found & Biflavonoids [20] \\
\hline $\begin{array}{l}\text { Searsia tenuinervis } \\
\text { (Engl.) Moffet }\end{array}$ & Shrub & Mufokosiana & $\begin{array}{l}\text { Leaves: extract drunk as menorrhagia } \\
\text { medicine [8] }\end{array}$ & Menorrhagia [1] & Antibacterial activity [21] \\
\hline \multicolumn{6}{|l|}{ Annonaceae } \\
\hline $\begin{array}{l}\text { Annona stenophylla } \\
\text { Engl. \& Diels }\end{array}$ & Shrub & Muroro & $\begin{array}{l}\text { Roots: paste applied on the boils; extract } \\
\text { drunk as chest pains and STI remedy; mixed } \\
\text { with roots of Securidaca longipedunculata } \\
\text { Fresen. and sprinkled around homestead } \\
\text { as snake repellent [8] }\end{array}$ & $\begin{array}{l}\text { Gonorrhoea, syphilis and } \\
\text { snake repellent [1] }\end{array}$ & Antioxidant activity [22] \\
\hline \multicolumn{6}{|l|}{ Apocynaceae } \\
\hline $\begin{array}{l}\text { Carissa bispinosa } \\
\text { (L.) Desf. ex Brenan }\end{array}$ & Shrub & Muruguru & $\begin{array}{l}\text { Roots: extract drunk as cough and } \\
\text { diarrhoea medicine [8] }\end{array}$ & None found & $\begin{array}{l}\text { Analgesic, antiviral and diuretic activities; lignans } \\
\text { and sesquiterpenes [23] }\end{array}$ \\
\hline $\begin{array}{l}\text { Carisa edulis } \\
\text { (Forssk.) Vahl }\end{array}$ & Shrub & Muruguru & $\begin{array}{l}\text { Roots: extract drunk as diarrhoea and } \\
\text { cough medicine [6] }\end{array}$ & $\begin{array}{l}\text { Cough }[1,24] \text {, chest pains } \\
\text { and pneumonia [1]; and } \\
\text { tuberculosis }{ }^{\#}[10]\end{array}$ & $\begin{array}{l}\text { Analgesic, antiviral and diuretic activities; lignans } \\
\text { and sesquiterpenes [23] }\end{array}$ \\
\hline $\begin{array}{l}{ }^{*} \text { Catharanthus roseus } \\
\text { (L.) G. Don }\end{array}$ & Herb & Chirindamatongo & $\begin{array}{l}\text { Roots: extract drunk as remedy for } \\
\text { stomach problems }[9,25]\end{array}$ & $\begin{array}{l}\text { Diarrhoea", dysentery" } \\
\text { and indigestion\# }[26]\end{array}$ & $\begin{array}{l}\text { Alkaloids, flavonoids, saponins, tannins and } \\
\text { triterpenes; antidiarrheal [27] and antidiabetic } \\
\text { [28] activities }\end{array}$ \\
\hline
\end{tabular}


Table 1 Medicinal plants used in south-central Zimbabwe (Continued)

\begin{tabular}{|c|c|c|c|c|c|}
\hline \multicolumn{6}{|l|}{ Asparagaceae } \\
\hline $\begin{array}{l}\text { Asparagus africanus } \\
\text { Lam. }\end{array}$ & Climber & Rukato & $\begin{array}{l}\text { Roots: extract drunk as diarrhoea and } \\
\text { pneumonia medicine and to dilate } \\
\text { birth canal [8] }\end{array}$ & Aid in child birth\# [29] & Analgesic and anti-inflammatory activities [30] \\
\hline $\begin{array}{l}\text { Sansevieria aethiopica } \\
\text { Thunb. }\end{array}$ & Herb & Zvikonje & $\begin{array}{l}\text { Leaves: leaf sap squeezed into the } \\
\text { painful ear [31] }\end{array}$ & Earache $^{\#}[32]$ & Antibacterial activity [33] \\
\hline $\begin{array}{l}\text { Sansevieria hyacinthoides } \\
\text { (L.) Druce }\end{array}$ & Herb & Masavamhanda & $\begin{array}{l}\text { Leaves or rhizomes: leaf or rhizome sap } \\
\text { given to child suffering from dehydration } \\
\text { [31]. Leaves: Leaf macerate given to colicky } \\
\text { infant; leaves used as dressing on sprained } \\
\text { ankle [31]. Rhizome: rhizome added to } \\
\text { non-alcoholic beverage taken by pregnant } \\
\text { women to prepare the birth canal and } \\
\text { prevent delivery complications; warm } \\
\text { rhizome extract given to a person with } \\
\text { aching tooth to keep in mouth for up to two } \\
\text { minutes before spitting out the mixture [31]. } \\
\text { Roots: extract drunk to dilate birth canal [9] }\end{array}$ & $\begin{array}{l}\text { Root used as baby } \\
\text { food }^{\#}[10]\end{array}$ & $\begin{array}{l}\text { Antibacterial, antioxidant [34] and } \\
\text { anti-inflammatory [12] properties. }\end{array}$ \\
\hline \multicolumn{6}{|l|}{ Asphodelaceae } \\
\hline $\begin{array}{l}\text { Aloe greatheadii } \\
\text { Schönland }\end{array}$ & Herb & Gavakava & $\begin{array}{l}\text { Leaves: extract drunk as constipation and } \\
\text { gonorrhoea medicine }[8]\end{array}$ & $\begin{array}{l}\text { Constipation and } \\
\text { gonorrhoea [1] }\end{array}$ & $\begin{array}{l}\text { Alkaloids, phenolic compounds and } \\
\text { antioxidant capacity [35] }\end{array}$ \\
\hline \multicolumn{6}{|l|}{ Asteraceae } \\
\hline Brachylaena discolor DC. & Herb & Mupasa & $\begin{array}{l}\text { Leaves: leaves chewed and juice swallowed } \\
\text { as remedy for ulcers [9] }\end{array}$ & $\begin{array}{l}\text { Enema to stop bleeding } \\
\text { of the stomach" }[36]\end{array}$ & Antidiabetic activity [28] \\
\hline Dicoma anomala Sond. & Herb & Chifumuro & $\begin{array}{l}\text { Bulb: extract drunk as remedy for } \\
\text { stomach upset [9] }\end{array}$ & $\begin{array}{l}\text { Remedy for all disease } \\
\text { (panacea) [1] }\end{array}$ & Sesquiterpene [37] \\
\hline $\begin{array}{l}\text { *Schkuhria pinnata } \\
\text { (Lam.) Kuntze ex Thell. }\end{array}$ & Herb & Ruhwahwa & $\begin{array}{l}\text { Whole plant: extract drunk as remedy for } \\
\text { stomach pains [9] }\end{array}$ & Stomach problems ${ }^{\#}[11]$ & Antibacterial [38] and anti-diarrhoeal activity [1 1] \\
\hline *Sonchus oleraceus L.; & Herb & Rurimirwemombe & $\begin{array}{l}\text { Leaves: extract drunk as remedy for } \\
\text { stomach problems [9] }\end{array}$ & $\begin{array}{l}\text { Anti-diarrhoeal }{ }^{\#} \text { and } \\
\text { digestive purgative }{ }^{\#} \text { [39] }\end{array}$ & $\begin{array}{l}\text { Alkaloids, flavonoids, phenols and saponins; } \\
\text { antioxidant and antibacterial [39] properties }\end{array}$ \\
\hline \multicolumn{6}{|l|}{ Burseraceae } \\
\hline $\begin{array}{l}\text { Commiphora marlothii } \\
\text { Engl. }\end{array}$ & Tree & Mupepe & Roots: extract drunk as STI medicine [9] & None found & Antibacterial activity [40] \\
\hline \multicolumn{6}{|l|}{ Celastraceae } \\
\hline $\begin{array}{l}\text { Gymnosporia buxifolia } \\
\text { (L.) Szyszyl. }\end{array}$ & Shrub & Chizhuzhu & $\begin{array}{l}\text { Leaves: leaves chewed and sap swallowed } \\
\text { as remedy for abdominal pains [9] }\end{array}$ & Painful menstruation ${ }^{\#}[41]$ & Antiplasmodial and anti-inflammatory activities [42] \\
\hline \multicolumn{6}{|l|}{ Chrysobalanaceae } \\
\hline $\begin{array}{l}\text { Parinari curatellifolia } \\
\text { Planch. ex Benth. }\end{array}$ & Tree & Muchakata & $\begin{array}{l}\text { Roots: extract drunk as constipation } \\
\text { medicine and teeth washed with root } \\
\text { decoction as remedy for toothache [8] }\end{array}$ & $\begin{array}{l}\text { Constipation }[1] \text { and } \\
\text { toothache } e^{\#}[1,10]\end{array}$ & $\begin{array}{l}\text { Antibacterial [13,43] and antimicrobial [43] } \\
\text { activities; alkaloids, flavonoids, phenol, saponins, } \\
\text { steroids, tannins and terpenes [43] }\end{array}$ \\
\hline
\end{tabular}


Table 1 Medicinal plants used in south-central Zimbabwe (Continued)

\begin{tabular}{|c|c|c|c|c|c|}
\hline \multicolumn{6}{|l|}{ Clusiaceae } \\
\hline $\begin{array}{l}\text { Garcinia buchananii } \\
\text { Baker }\end{array}$ & Tree & Mutunduru & $\begin{array}{l}\text { Bark: extract drunk to reduce birth canal [9]. } \\
\text { Fruits: ripe fruits are eaten as aphrodisiac [9] }\end{array}$ & $\begin{array}{l}\text { Aphrodisiac and to } \\
\text { reduce birth canal [1] }\end{array}$ & $\begin{array}{l}\text { Anthraquinones and cytotoxicity activity }[44,45] \text {, } \\
\text { phenolics, steroids and tannins }[46]\end{array}$ \\
\hline \multicolumn{6}{|l|}{ Cucurbitaceae } \\
\hline Cucumis anguria $\mathrm{L}$. & Herb & Muchacha & $\begin{array}{l}\text { Fruits: pieces of fruit left around } \\
\text { homestead as snake repellent [8] }\end{array}$ & Antifeedant [47] & Larvicidal activity and triterpenoids [47] \\
\hline \multicolumn{6}{|l|}{ Cyperaceae } \\
\hline $\begin{array}{l}\text { Coleochloa setifera } \\
\text { (Ridl.) Gilly }\end{array}$ & Herb & Rufuri & $\begin{array}{l}\text { Roots: root powder taken as a remedy } \\
\text { for pneumonia [12] }\end{array}$ & None found & None found \\
\hline \multicolumn{6}{|l|}{ Ebenaceae } \\
\hline Diospyros lycioides Desf. & Shrub & Musumadombo & $\begin{array}{l}\text { Roots: extract drunk as remedy for } \\
\text { infertility in women [8] }\end{array}$ & Infertility in women $\#[1,10]$ & $\begin{array}{l}\text { Antibacterial activity [48]; lupeol and ursolic acid } \\
\text { [49] }\end{array}$ \\
\hline $\begin{array}{l}\text { Diospyros mespiliformis } \\
\text { Hochst. ex A.DC. }\end{array}$ & Tree & Musuma & $\begin{array}{l}\text { Roots: extract drunk as abdominal pains } \\
\text { medicine [8] }\end{array}$ & Body and heart pains [1] & $\begin{array}{l}\text { Antimicrobial activity; saponins, steroids, } \\
\text { tannins and triterpene [50] }\end{array}$ \\
\hline $\begin{array}{l}\text { Euclea crispa (Thunb.) } \\
\text { Sond. ex Gürke }\end{array}$ & Shrub & Muvhinji & Roots: extract drunk as cough medicine [8] & Cough [1] & Antibacterial activity [51] \\
\hline Euclea divinorum Hiern & Shrub & Mushangura & $\begin{array}{l}\text { Roots: extract drunk as diarrhoea } \\
\text { medicine [8] }\end{array}$ & $\begin{array}{l}\text { Diarrhoea [1]; and troubled } \\
\text { and noisy stamach" }{ }^{\# 10]}\end{array}$ & Antimicrobial activity [44] \\
\hline \multicolumn{6}{|l|}{ Euphorbiaceae } \\
\hline $\begin{array}{l}\text { Androstachys johnsonii } \\
\text { Prain }\end{array}$ & Tree & Musimbiti & Roots: extract drunk as aphrodisiac [9] & None found & $\begin{array}{l}\text { Antibacterial [52], antimicrobial and } \\
\text { antifungal [53] activities }\end{array}$ \\
\hline $\begin{array}{l}\text { Bridelia cathartica } \\
\text { G. Bertol. }\end{array}$ & Shrub & Mutsvoritsvoto & $\begin{array}{l}\text { Roots: extract drunk as remedy for infertility } \\
\text { in men [8] }\end{array}$ & Infertility in men [1] & $\begin{array}{l}\text { Anthocyanins, flavonoids and tannins [54]; } \\
\text { antibacterial [54] and antimalarial [55] activities }\end{array}$ \\
\hline Bridelia mollis Hutch. & Shrub & Mutuzvidzembwa & Roots: extract drunk as cough medicine [8] & None found & None found \\
\hline $\begin{array}{l}\text { Flueggea virosa } \\
\text { (Roxb. ex Willd.) Voigt }\end{array}$ & Shrub & Mushagahuwe & $\begin{array}{l}\text { Roots: extract drunk as pneumonia } \\
\text { medicine and drunk before sexual } \\
\text { intercourse as a contraceptive; dried root } \\
\text { powder applied to bitten part as snake } \\
\text { antidote and root powder applied on } \\
\text { wounds [8] }\end{array}$ & $\begin{array}{l}\text { Contraceptive, pneumonia } \\
\text { and snake antidote [1] }\end{array}$ & $\begin{array}{l}\text { Alkaloids, securinine and triterpenes; antifungal, } \\
\text { antimalarial, antimicrobial and antioxidant } \\
\text { activities [56-59] }\end{array}$ \\
\hline $\begin{array}{l}\text { Macaranga capensis } \\
\text { (Baill.) Benth. ex Sim }\end{array}$ & Tree & Musvosve & Roots: extract drunk as aphrodisiac[9] & Male impotence ${ }^{\#}[60]$ & Antibacterial activity [60] \\
\hline *Ricinus communis L. & Herb & Mupfuta & $\begin{array}{l}\text { Roots: teeth washed with root decoction } \\
\text { as remedy for toothache [8]. Seed: oil } \\
\text { applied on sore eyes [8] }\end{array}$ & $\begin{array}{l}\text { Sore eyes }[1] \text { and } \\
\text { toothache } e^{\#}[1,10]\end{array}$ & $\begin{array}{l}\text { Anti-inflammatory, antiarthritic [61,62], } \\
\text { anti-oxidant, antiulcer, antidiabetic, } \\
\text { antifertility and antimicrobial [63] properties }\end{array}$ \\
\hline $\begin{array}{l}\text { Spirostachys africana } \\
\text { Sond. }\end{array}$ & Tree & Munhiti & $\begin{array}{l}\text { Roots: root powder mixed with porridge } \\
\text { as remedy for venereal infections [9] }\end{array}$ & None found & $\begin{array}{l}\text { Flavonoids and gallotannins [64], phenolic } \\
\text { and antioxidant activity [65] }\end{array}$ \\
\hline
\end{tabular}


Table 1 Medicinal plants used in south-central Zimbabwe (Continued)

\begin{tabular}{|c|c|c|c|c|c|}
\hline \multicolumn{6}{|l|}{ Fabaceae sensu lato } \\
\hline Acacia karroo Hayne & Tree & Muvunga & $\begin{array}{l}\text { Root: extract drunk as convulsions } \\
\text { remedy [8]; aphrodisiac, gonorrhoea } \\
\text { and syphilis [9] }\end{array}$ & $\begin{array}{l}\text { Aphrodisiac, convulsions, } \\
\text { gonorrhoea and syphilis [1] }\end{array}$ & $\begin{array}{l}\text { Anti-inflammatory, analgesic [66], antibacterial } \\
\text { [67] and antimicrobial [66] activities }\end{array}$ \\
\hline $\begin{array}{l}\text { Albizia antunesiana } \\
\text { Harms }\end{array}$ & Tree & Muriranyenze & $\begin{array}{l}\text { Bark: extract drunk as constipation } \\
\text { remedy [8]. Leaves: extract drunk as } \\
\text { purgative remedy [8]. Roots: extract } \\
\text { drunk as aphrodisiac, diarrhoea, } \\
\text { gonorrhoea and remedy for infertility } \\
\text { in women [8] }\end{array}$ & $\begin{array}{l}\text { Aphrodisiac }[1,24] \text {, } \\
\text { gonorrhoea, } \\
\text { infertility in women and } \\
\text { as purgative }[1]\end{array}$ & Anthelmintic activity [15] \\
\hline $\begin{array}{l}\text { Brachystegia boehmii } \\
\text { Taub. }\end{array}$ & Tree & Mupfuti & Bark: extract drunk as STI medicine [8] & None found & Antibacterial activity [13] \\
\hline Cassia abbreviata Oliv. & Shrub & Muremberembe & $\begin{array}{l}\text { Roots: extract drunk as abortion, } \\
\text { aphrodisiac, constipation, diarrhoea } \\
\text { and gonorrhoea medicine [8] }\end{array}$ & $\begin{array}{l}\text { Abortion, aphrodisiac } \\
\text { constipation diarrhoea } \\
\text { and gonorrhoea [1] }\end{array}$ & $\begin{array}{l}\text { Anthraquinones, triterpenoids [68], antibacterial } \\
\text { [55], antimalarial [69] and antiviral [70] activities }\end{array}$ \\
\hline $\begin{array}{l}\text { Dalbergia melanoxylon } \\
\text { Guill. \& Perr. }\end{array}$ & Shrub & Mugwiti & $\begin{array}{l}\text { Leaves: dried leaves smoked as cigarette } \\
\text { to treat asthma [9] }\end{array}$ & $\begin{array}{l}\text { Bronchitis" and inflammation } \\
\text { in throat" }[71]\end{array}$ & Antimicrobial activity [72] \\
\hline $\begin{array}{l}\text { Elephantorrhiza goetzei } \\
\text { (Harms) Harms }\end{array}$ & Shrub & Ntorani & $\begin{array}{l}\text { Roots: extract drunk as abdominal pains, } \\
\text { diarrhoea and gonorrhoea medicine; and } \\
\text { mixed with roots of Piliostigma thonningii } \\
\text { (Schumach.) Milne-Redh. as bilharzia } \\
\text { (schistosomiasis) medicine [8] }\end{array}$ & $\begin{array}{l}\text { Abdominal pains }[1,24] \text {, } \\
\text { bilharzias, diarrhoea } \\
\text { and gonorrhoea [1] }\end{array}$ & Anthelmintic activity [15] and stilbenes [73] \\
\hline $\begin{array}{l}\text { Erythrina abyssinica } \\
\text { Lam. ex DC. }\end{array}$ & Tree & Mutiti & $\begin{array}{l}\text { Bark: extract drunk as backache medicine [8]. } \\
\text { Roots: wounds washed with root extract [8] }\end{array}$ & $\begin{array}{l}\text { Backache and wounds in } \\
\text { mouth [1] }\end{array}$ & $\begin{array}{l}\text { Antibacterial [74,75], antifungal [74] and } \\
\text { cytotoxic activities [76] }\end{array}$ \\
\hline Indigofera setiflora Baker & Herb & Ruvavashuro & Roots: extract drunk as diarrhoea medicine [9] & None found & None found \\
\hline $\begin{array}{l}\text { Peltophorum africanum } \\
\text { Sond. }\end{array}$ & Shrub & Muzeze & $\begin{array}{l}\text { Bark, leaves or root: extract drunk as } \\
\text { syphilis medicine [8]. Roots: extract drunk as } \\
\text { diarrhoea and STI medicine, root extract used } \\
\text { as eye drops for sore eyes and teeth washed } \\
\text { with root decoction as remedy for } \\
\text { toothache [8] }\end{array}$ & $\begin{array}{l}\text { Diarrhoea and toothache } \\
{[1] \text {, panacea }[24] \text { and }} \\
\text { venereal diseases }{ }^{\#}[1,10]\end{array}$ & Antibacterial activity $[52,77]$ \\
\hline $\begin{array}{l}\text { Piliostigma thonningii } \\
\text { (Schumach.) } \\
\text { Milne-Redh. }\end{array}$ & Tree & Musekesa & $\begin{array}{l}\text { Bark, leaves or root: extract drunk as cough } \\
\text { medicine [8]. Leaves:extract drunk as } \\
\text { menorrhagia medicine [8]. Roots: mixed with } \\
\text { roots of Elephantorrhiza goetzei as bilharzia } \\
\text { (schistosomiasis) medicine [8] }\end{array}$ & Cough and menorrhagia [1] & $\begin{array}{l}\text { Alkaloids, flavonoids, saponins and tannins; } \\
\text { antibacterial [16], antimicrobial and } \\
\text { antioxidant [78] activities }\end{array}$ \\
\hline $\begin{array}{l}\text { Pterocarpus } \\
\text { angolensis DC. }\end{array}$ & Tree & Mubvamaropa & $\begin{array}{l}\text { Bark: extract dropped into ear as earache } \\
\text { medicine, extract drunk as remedy for } \\
\text { menorrhagia [8]. Roots: extract drunk as } \\
\text { remedy for infertility in women [8]. } \\
\text { Sap: dropped into sore eyes [8] }\end{array}$ & $\begin{array}{l}\text { Infertility in women and } \\
\text { sore eyes }[1] \text {; and } \\
\text { menorrhagia }[1,10]\end{array}$ & Antibacterial and cytotoxicity activities [79] \\
\hline \multicolumn{6}{|l|}{ Flacourtiaceae } \\
\hline $\begin{array}{l}\text { Flacourtia indica } \\
\text { (Burm. f.) Merr. }\end{array}$ & Shrub & Munhunguru & $\begin{array}{l}\text { Leaves: leaves browsed by mouth as } \\
\text { diarrhoea medicine [8] }\end{array}$ & Diarrhoea [1] & $\begin{array}{l}\text { Antibacterial, anti-inflammatory, antimicrobial, } \\
\text { antioxidant and antimalarial activities [80] }\end{array}$ \\
\hline
\end{tabular}


Table 1 Medicinal plants used in south-central Zimbabwe (Continued)

\begin{tabular}{|c|c|c|c|c|c|}
\hline \multicolumn{6}{|l|}{ Hypoxidaceae } \\
\hline $\begin{array}{l}\text { Hypoxis obtusa } \\
\text { Ker Gawl. }\end{array}$ & Herb & Nhindiri & $\begin{array}{l}\text { Bulb: bulb chewed and sap swallowed } \\
\text { as remedy for abdominal pains [9] }\end{array}$ & Abdominal pains [1] & Hypoxoside [81] and obtusaside [82] \\
\hline \multicolumn{6}{|l|}{ Kirkiaceae } \\
\hline Kirkia acuminata Oliv. & Tree & Mubvumira & $\begin{array}{l}\text { Bark: extract drunk as diarrhoea, cholera, } \\
\text { dysentery and constipation medicine [9]. } \\
\text { Fruits: fruit juice applied to bitten part as } \\
\text { snake antidote and fruit juice applied } \\
\text { on wounds [8] }\end{array}$ & Diarrhoea and wounds [1] & Antibacterial activity [61] \\
\hline \multicolumn{6}{|l|}{ Lamiaceae } \\
\hline $\begin{array}{l}\text { Hoslundia } \\
\text { opposita Vahl }\end{array}$ & Herb & Hwahwa hwe shiri & $\begin{array}{l}\text { Leaves: extract dropped into eyes as } \\
\text { cataract medicine [8] }\end{array}$ & Cataract [1] & $\begin{array}{l}\text { Alkaloids, flavonoids, saponins, tannins and } \\
\text { triterpenes }[83,84] \text { and antimicrobial activity }[85]\end{array}$ \\
\hline $\begin{array}{l}\text { Leonotis leonurus } \\
\text { (L.) R.Br. }\end{array}$ & Herb & Mutodzvo & $\begin{array}{l}\text { Leaves: leaves chewed and sap swallowed } \\
\text { as remedy for ulcers [9] }\end{array}$ & Sores" and haemorrhoids" ${ }^{\#}$ [84] & $\begin{array}{l}\text { Anti-inflammatory, cytotoxic and } \\
\text { hepatoprotective activities [86] }\end{array}$ \\
\hline $\begin{array}{l}\text { Vitex payos } \\
\text { (Lour.) Merr. }\end{array}$ & Tree & Mutsvubvu & $\begin{array}{l}\text { Leaves: leaves burnt and smoke inhaled } \\
\text { as cough medicine }[8,9]\end{array}$ & None found & Larvicidal activity [87] \\
\hline \multicolumn{6}{|l|}{ Loganiaceae } \\
\hline $\begin{array}{l}\text { Strychnos } \\
\text { cocculoides Bak. }\end{array}$ & Tree & Muzumwi & $\begin{array}{l}\text { Roots: extract drunk as abdominal pains, } \\
\text { aphrodisiac, gonorrhoea, infertility in men } \\
\text { and sore throat remedy [8] }\end{array}$ & $\begin{array}{l}\text { Abdominal pains and } \\
\text { infertility [1] }\end{array}$ & Antimalarial activity [88] \\
\hline $\begin{array}{l}\text { Strychnos } \\
\text { madagascariensis } \\
\text { Poir. }\end{array}$ & Tree & Mukwakwa & $\begin{array}{l}\text { Roots: extract used as eye drops for } \\
\text { sore eyes [8] }\end{array}$ & None found & None found \\
\hline Strychnos spinosa Lam. & Tree & Mutamba & $\begin{array}{l}\text { Roots: extract drunk as remedy for } \\
\text { abdominal pains and gonorrhoea [8]. } \\
\text { Fruits: extract drunk as remedy for } \\
\text { gonorrhoea and genital warts [9] }\end{array}$ & Abdominal pains [1] & Sterols and triterpenoids [89] \\
\hline \multicolumn{6}{|l|}{ Malvaceae } \\
\hline $\begin{array}{l}\text { Azanza garckeana } \\
\text { (F. Hoffm.) Exell \& Hillc. }\end{array}$ & Tree & Mutohwe & $\begin{array}{l}\text { Roots: extract dropped into the ear as } \\
\text { medicine for earache [8] }\end{array}$ & Earache $^{\#}[90]$ & Antimalarial activity [69] \\
\hline \multicolumn{6}{|l|}{ Meliaceae } \\
\hline $\begin{array}{l}\text { Ekebergia benguelensis } \\
\text { Welw. ex C.DC. }\end{array}$ & Tree & Mudyavarungu & $\begin{array}{l}\text { Bark: extract drunk as remedy for infertility } \\
\text { in men [8]. } \\
\text { Roots: extract drunk as dysmenorrhea } \\
\text { medicine [8] }\end{array}$ & $\begin{array}{l}\text { Dysmenorrhea and } \\
\text { infertility in men [1] }\end{array}$ & Stilbenes [91] and triperpenes [92] \\
\hline $\begin{array}{l}\text { Entandrophragma } \\
\text { caudatum (Sprague) } \\
\text { Sprague }\end{array}$ & Tree & Mubanana & $\begin{array}{l}\text { Fruits: burnt fruit peels mixed with } \\
\text { vaseline and applied } \\
\text { to area affected with genital warts [9] }\end{array}$ & None found & Limonoids [93] \\
\hline \multicolumn{6}{|l|}{ Moraceae } \\
\hline $\begin{array}{l}\text { Ficus ingens } \\
\text { (Miq.) Miq. }\end{array}$ & Tree & Mushavhi & Roots: extract drunk as cough medicine [8] & Fever ${ }^{\#}[94]$ & Anti-inflammatory and analgesic properties [95] \\
\hline
\end{tabular}


Table 1 Medicinal plants used in south-central Zimbabwe (Continued)

\begin{tabular}{|c|c|c|c|c|c|}
\hline Ficus sur Forssk. & Tree & Muonde & $\begin{array}{l}\text { Roots: extract drunk as diarrhoea and } \\
\text { syphilis medicine [8] }\end{array}$ & Diarrhoea in infants [1] & $\begin{array}{l}\text { Antibacterial, anti-inflammatory, antimalarial } \\
\text { and anti-ulcer activities }[96,97]\end{array}$ \\
\hline Ficus sycomorus L. & Tree & Muonde & Roots: extract drunk as cough medicine [8] & $\begin{array}{l}\text { Tuberculosis", cold }{ }^{\#} \text { and } \\
\text { other chest problems }{ }^{\#}[10]\end{array}$ & $\begin{array}{l}\text { Antibacterial activity [98], alkaloids, saponins } \\
\text { and tannins [99] }\end{array}$ \\
\hline \multicolumn{6}{|l|}{ Moringaceae } \\
\hline *Moringa oleifera Lour & Shrub & Moringa & $\begin{array}{l}\text { Leaves: extract drunk as diarrhoea } \\
\text { medicine [8]. Roots: teeth washed with root } \\
\text { decoction as remedy for toothache [8] }\end{array}$ & Digestive disorders [100] & $\begin{array}{l}\text { Alkaloids and flavonoids, anti-inflammatory, } \\
\text { antioxidant, antimicrobial, antifertility and } \\
\text { anticancer [101] activities }\end{array}$ \\
\hline \multicolumn{6}{|l|}{ Myrothamnaceae } \\
\hline $\begin{array}{l}\text { Myrothamnus } \\
\text { flabellifolius } \\
\text { Welw. }\end{array}$ & Shrub & Rufandichimuka & $\begin{array}{l}\text { Leaves and twigs: leaves and twigs boiled } \\
\text { and drunk as remedy for cold [9] }\end{array}$ & $\begin{array}{l}\text { Cold }{ }^{\#} \text { and other chest } \\
\text { complaints } s^{\#}[1,10]\end{array}$ & $\begin{array}{l}\text { Alkaloids, flavanoids, phenolics, saponins, } \\
\text { steroids and tannins [102], antidiabetic } \\
\text { [103] and antimicrobial [104] activities }\end{array}$ \\
\hline \multicolumn{6}{|l|}{ Myrtaceae } \\
\hline $\begin{array}{l}{ }^{*} \text { Eucalyptus } \\
\text { camaldulensis } \\
\text { Dehnh }\end{array}$ & Tree & Mugamutiri & $\begin{array}{l}\text { Leaves: extract drunk with Citrus limon fruits } \\
\text { and Psidium guajava L. leaves as cough, flu } \\
\text { and fever medicine [8] }\end{array}$ & Sore throat ${ }^{\#}[105]$ & Antiproliferative [106] and antimicrobial [107] activitie \\
\hline *Psidium guajava $\mathrm{L}$. & Shrub & Mugwavha & $\begin{array}{l}\text { Leaves: extract drunk with Citrus limon fruits } \\
\text { and Eucalyptus camaldulensis leaves as cough, } \\
\text { flu and fever medicine [8]; infusion of drunk } \\
\text { or taken as an enema for diarrhoea [9] }\end{array}$ & $\begin{array}{l}\text { Fever }[1] \text {; cough"\# }[1,11,85] \\
\text { and diarrhoea\# }[11,85]\end{array}$ & $\begin{array}{l}\text { Anti-diarrhoeal [108,109], antibacterial [110,111], } \\
\text { narcotic [109] and antioxidant [112] properties }\end{array}$ \\
\hline $\begin{array}{l}\text { Syzygium cordatum } \\
\text { Hochst. ex C. Krauss }\end{array}$ & Tree & Mukute & $\begin{array}{l}\text { Bark: extract drunk as tuberculosis } \\
\text { medicine [9] }\end{array}$ & Cold $^{\#}$ and fever ${ }^{\#}[10]$ & Antibacterial [52] and antifungal [64] activities \\
\hline \multicolumn{6}{|l|}{ Ochnaceae } \\
\hline Ochna pulchra Hook.f. & Shrub & Munimu & $\begin{array}{l}\text { Leaves: leaf sap taken as remedy for } \\
\text { stomach problems [9] }\end{array}$ & Diarrhoea [1] & Antibacterial activity [113] \\
\hline \multicolumn{6}{|l|}{ Olacaceae } \\
\hline Ximenia americana L. & Shrub & Mutengeni & $\begin{array}{l}\text { Leaves: extract drunk as backache } \\
\text { medicine [8] }\end{array}$ & Abdominal pains ${ }^{\#}[114]$ & Antibacterial [64] and antioxidant [114] activities \\
\hline Ximenia caffra Sond. & Shrub & Munhengeni & $\begin{array}{l}\text { Leaves: extract drunk as backache } \\
\text { medicine [8]. Roots: extract drunk as } \\
\text { aphrodisiac, diarrhoea, venereal diseases; root } \\
\text { powder applied on wounds [8] }\end{array}$ & $\begin{array}{l}\text { Diarrhoea and infertility [1]; } \\
\text { and venereal diseases }{ }^{\#}[1,10]\end{array}$ & $\begin{array}{l}\text { Flavonoids, phenolic and tannins; } \\
\text { and antimicrobial activity [115] }\end{array}$ \\
\hline \multicolumn{6}{|l|}{ Pedaliaceae } \\
\hline $\begin{array}{l}\text { Dicerocaryum } \\
\text { zanguebarium } \\
\text { (Klotzsch) Abels }\end{array}$ & Herb & Ruredzo & $\begin{array}{l}\text { Whole plant: plant foam inserted into } \\
\text { vagina to dilate birth canal [9] }\end{array}$ & $\begin{array}{l}\text { To dilate birth canal [1] and } \\
\text { expulsion of placenta" }[10]\end{array}$ & Cytotoxic activity [116] \\
\hline \multicolumn{6}{|l|}{ Polygalaceae } \\
\hline $\begin{array}{l}\text { Securidaca } \\
\text { longepedunculata } \\
\text { Fresen. }\end{array}$ & Shrub & Mufufu & $\begin{array}{l}\text { Roots: extract drunk as epilepsy medicine } \\
\text { and mixed with roots of Annona stenophylla } \\
\text { and sprinkled around homestead as } \\
\text { snake repellent [8] }\end{array}$ & $\begin{array}{l}\text { Epilepsy [1] and snake } \\
\text { repellent [24] }\end{array}$ & $\begin{array}{l}\text { Analgesic, anti-inflammatory, hypoglycaemic } \\
{[117] \text { and antimalarial [88] activities }}\end{array}$ \\
\hline
\end{tabular}


Table 1 Medicinal plants used in south-central Zimbabwe (Continued)

\begin{tabular}{|c|c|c|c|c|c|}
\hline \multicolumn{6}{|l|}{ Pteridaceae } \\
\hline Pellaea sp. & Fern & Mudziwebwe & $\begin{array}{l}\text { Leaves and roots: leaves and roots burnt } \\
\text { and smoke inhaled as remedy for chest } \\
\text { pains [9] }\end{array}$ & None found & None found \\
\hline \multicolumn{6}{|l|}{ Rhamnaceae } \\
\hline $\begin{array}{l}\text { Berchemia discolor } \\
\text { (Klotzsch) Hemsl. }\end{array}$ & Tree & Nyii & $\begin{array}{l}\text { Roots: extract drunk as abdominal } \\
\text { pains medicine [8] }\end{array}$ & General body pains [1] & Antimicrobial activity [118] and flavonoids [119] \\
\hline $\begin{array}{l}\text { Ziziphus mucronata } \\
\text { Willd. }\end{array}$ & Tree & Muchecheni & $\begin{array}{l}\text { Fruits and leaves: powder applied on } \\
\text { boils [8]. Roots: extract drunk as abdominal } \\
\text { pains, infertility in women medicine and } \\
\text { root } \\
\text { powder applied on wounds [8] }\end{array}$ & $\begin{array}{l}\text { Skin infections and wounds } \\
{[1] ; \text { body pains } s^{\#} \text { and infertility }} \\
\text { in women }{ }^{\#}[10] \text {; boils", sores } \\
\text { and swellings }{ }^{\#}[11]\end{array}$ & Anthelmintic [15] and antimicrobial [120] activities \\
\hline \multicolumn{6}{|l|}{ Rosaceae } \\
\hline *Prunus persica L. & Tree & Mupichisi & $\begin{array}{l}\text { Leaves: extract drunk as diarrhoea } \\
\text { medicine [22] }\end{array}$ & None found & $\begin{array}{l}\text { Antimicrobial, antioxidant [121]; anti-tumour promoter } \\
\text { and anti-Oketsu syndrome [122] effects }\end{array}$ \\
\hline \multicolumn{6}{|l|}{ Rubiaceae } \\
\hline $\begin{array}{l}\text { Crossopteryx febrifuga } \\
\text { (Afzel. ex G. Don) } \\
\text { Benth. }\end{array}$ & Shrub & Mukomberwa & $\begin{array}{l}\text { Bark: added to porridge as remedy for } \\
\text { diarrhoea and dysentery }[9]\end{array}$ & $\begin{array}{l}\text { Diarrhoea } \# \text { and } \\
\text { dysentery }{ }^{\# 123]}\end{array}$ & $\begin{array}{l}\text { Alkaloids, flavonoids, saponins, steroids, tannins } \\
\text { and terpenoids [124], anti-inflammatory and } \\
\text { antimicrobial activities [125] }\end{array}$ \\
\hline $\begin{array}{l}\text { Vangueria infausta } \\
\text { Burch. }\end{array}$ & Tree & Mudzvirungombe & $\begin{array}{l}\text { Roots: extract drunk as diarrhoea } \\
\text { medicine [8] }\end{array}$ & Diarrhoea [1] & $\begin{array}{l}\text { Flavonoids, antibacterial [126,127], } \\
\text { antimalarial [88] and antifungal [126] activities }\end{array}$ \\
\hline \multicolumn{6}{|l|}{ Rutaceae } \\
\hline $\begin{array}{l}{ }^{*} \text { Citrus limon } \\
\text { (L.) Burm. f. }\end{array}$ & Tree & Mulemoni & $\begin{array}{l}\text { Fruit: extract of fruit, Eucalyptus } \\
\text { camaldulensis and Psidium guajava leaves } \\
\text { drunk as cough, flu and fever medicine [8] }\end{array}$ & $\begin{array}{l}\text { Throat infections }{ }^{\#} \\
\text { and tonsil }\left.\right|^{\#}[128]\end{array}$ & $\begin{array}{l}\text { Analgesic, intestinal mucosa protector } \\
\text { and antiseptic [128] }\end{array}$ \\
\hline \multicolumn{6}{|l|}{ Solanaceae } \\
\hline *Nicotiana tobacum L. & Herb & Fodya & Leaves: snuff applied on wounds [8] & Wounds [1] & $\begin{array}{l}\text { Cytotoxic activity [129] and steroidal glycosides } \\
\text { [130] }\end{array}$ \\
\hline *Solanum incanum L. & Shrub & Nhundurwa & $\begin{array}{l}\text { Fruits: child bathed with fruit macerate } \\
\text { as remedy for scabies [9] }\end{array}$ & $\begin{array}{l}\text { Rash", ringworm }{ }^{\#} \text {, } \\
\text { skin infections }{ }^{\#} \\
\text { and warts }{ }^{\#}[131]\end{array}$ & $\begin{array}{l}\text { Saponins; antibacterial, cytotoxicity [16], } \\
\text { antioxidant and cytoprotective [132] activities }\end{array}$ \\
\hline \multicolumn{6}{|l|}{ Tiliaceae } \\
\hline Corchorus tridens $\mathrm{L}$. & Herb & Derere & $\begin{array}{l}\text { Roots: extract drunk as backache } \\
\text { medicine [8] }\end{array}$ & None found & Flavonoids and microbial activity [133] \\
\hline Grewia bicolor Juss. & Shrub & Mutewa & $\begin{array}{l}\text { Roots: extract drunk as diarrhoea and } \\
\text { gonorrhoea medicine [8] }\end{array}$ & Diarrhoea\# [10] & Alkaloids, triterpenoids and antibacterial activity [134] \\
\hline Grewia flavescens Juss. & Shrub & Mubhubhunu & $\begin{array}{l}\text { Roots: extract drunk as menorrhagia } \\
\text { medicine [8] }\end{array}$ & $\begin{array}{l}\text { Inducing labour", } \\
\text { infertility } \text { and } \\
\text { impotence }{ }^{\#}[10]\end{array}$ & Triterpenoids [101] \\
\hline Grewia monticola Sond. & Shrub & Mutewa & & Diarrhoea [1] & None found \\
\hline
\end{tabular}


Table 1 Medicinal plants used in south-central Zimbabwe (Continued)

\begin{tabular}{|c|c|c|c|c|c|}
\hline & & & $\begin{array}{l}\text { Roots: extract drunk as diarrhoea } \\
\text { medicine [8] }\end{array}$ & & \\
\hline \multicolumn{6}{|l|}{ Urticaceae } \\
\hline Pouzolzia mixta Solms & Shrub & Munanzwa & $\begin{array}{l}\text { Roots: extract instilled into the vagina to } \\
\text { dilate birth canal; extract drunk as STI } \\
\text { medicine and root powder applied } \\
\text { on wounds [8] }\end{array}$ & $\begin{array}{l}\text { To dilate birth canal } \\
{[1,24] \text { and venereal }} \\
\text { diseases }[1]\end{array}$ & Antibacterial activity [52] \\
\hline \multicolumn{6}{|l|}{ Verbenaceae } \\
\hline *Lantana camara L. & Shrub & Mbarambati & $\begin{array}{l}\text { Leaves: leaf sap applied on body parts } \\
\text { infected with ring worm [9] }\end{array}$ & Eye injuries ${ }^{\#}[10]$ & $\begin{array}{l}\text { Antibacterial [13] and antimalarial [88] } \\
\text { activities; flavonoids and triterpenes [135. }\end{array}$ \\
\hline $\begin{array}{l}\text { Lippia javanica } \\
\text { (Burm.f.) Spreng. }\end{array}$ & Shrub & Zimbani & $\begin{array}{l}\text { Leaves and twigs: boiled leaves and twigs } \\
\text { drunk as remedy for cough and cold [9] }\end{array}$ & Cold and cough $[1,10,24]$ & Antimicrobial activity [136] \\
\hline \multicolumn{6}{|l|}{ Vitaceae } \\
\hline $\begin{array}{l}\text { Ampelocissus africanus } \\
\text { (Lour.) Merr. }\end{array}$ & Climber & Muzambiringa & $\begin{array}{l}\text { Roots: extract drunk as diarrhoea } \\
\text { medicine [8] }\end{array}$ & Stomach troubles" ${ }^{\# 137]}$ & Antibacterial activity [138] \\
\hline $\begin{array}{l}\text { Ampelocissus obtusata } \\
\text { (Welw. ex Baker) Planch. }\end{array}$ & Climber & Muzambiringa & $\begin{array}{l}\text { Roots: extract drunk as diarrhoea } \\
\text { medicine [8] }\end{array}$ & $\begin{array}{l}\text { Gastro-intestinal } \\
\text { complaints }^{\#}[137]\end{array}$ & Antibacterial activity [138] \\
\hline \multicolumn{6}{|l|}{ Zingiberaceae } \\
\hline $\begin{array}{l}\text { *Zingiber officinale } \\
\text { Roscoe }\end{array}$ & Herb & Tsangamidzi & $\begin{array}{l}\text { Roots: roots chewed and swallowed as } \\
\text { remedy for stomach pains [9] }\end{array}$ & Digestive disorders ${ }^{\#}$ [139] & $\begin{array}{l}\text { Antimicrobial [16], anti-inflammatory, } \\
\text { immuno-modulatory and anti-emetic } \\
\text { [140] activities }\end{array}$ \\
\hline
\end{tabular}

An asterisk (*) indicates that the taxon is known or believed to be exotic to Zimbabwe and hatch (\#) indicates similar use(s) in other countries reported from literature. 
Table 2 Families with the largest number of medicinal plants (more than 2 species) in south-central Zimbabwe

\begin{tabular}{lll}
\hline Family & Number of medicinal plants & $\%$ \\
\hline Fabaceae sensu lato & 11 & 11.8 \\
Anacardiaceae & 9 & 9.7 \\
Euphorbiaceae & 7 & 9.5 \\
Asteraceae & 4 & 4.3 \\
Ebenaceae & 4 & 4.3 \\
Tiliaceae & 4 & 4.3 \\
Apocynaceae & 3 & 3.2 \\
Asparagaceae & 3 & 3.2 \\
Lamiaceae & 3 & 3.2 \\
Loganiaceae & 3 & 3.2 \\
Moraceae & 3 & 3.2 \\
Myrtaceae & 3 & 3.2 \\
Meliaceae & 2 & 2.2 \\
Olacaceae & 2 & 2.2 \\
Rhamnaceae & 2 & 2.2 \\
Rubiaceae & 2 & 2.2 \\
Solanaceae & 2 & 2.2 \\
Verbenaceae & 2 & 2.2 \\
Vitaceae & 2 & 2.2 \\
\hline
\end{tabular}

(4 species each). Fabaceae, Anacardiaceae and Euphorbiaceae families have the highest number of species used as herbal medicines probably because these are large families characterized by several species. The rest of the families were represented by one species each (Table 1). The genera with highest number of species were Ficus, Grewia, Searsia and Strychnos with 3 species each.

\section{Growth habit and parts used}

Trees and shrubs (38\% each) are the primary sources of the medicinal plant species in south-central Zimbabwe, followed by herbs (21\%) and climbers (3\%) (Figure 2A). Extensive use of trees and shrubs in south-central Zimbabwe in preparation of herbal medicines might be linked to their availability throughout the year as they are relatively drought resistant and are not affected by seasonal variations [8]. The plant parts used for making herbal preparations were the bark, bulbs, fibre, fruits, leaves, rhizomes, roots, sap, seeds, twigs and whole plant. The roots were the most frequently used (61.3\%), followed by leaves (32.3\%), bark (12.9\%), fruits (9.7\%), sap (5.4\%), bulbs, twigs and whole plant (2.2\% each), fibre, seeds and rhizomes (1.1\% each) (Figure 2B). The use of whole plant as remedy was administered for herbaceous plant species (Table 1). However, harvesting of roots of herbaceous plants for medicinal purposes is not sustainable as it threatens the survival of the same plants used to treat human ailments in south-central Zimbabwe. It is well recognized by conservationists that medicinal plants primarily valued for their root parts and those which are intensively harvested for their bark often tend to be the most threatened by over-exploitation [141].

\section{Ailments and diseases treated and herbal preparation} The majority of the plant species used (61.3\%) had a single therapeutic use, with 19 species $(20.4 \%)$ used in the treatment of two ailments, 6 species (6.5\%) treating three ailments, 5 species $(5.4 \%)$ treating four ailments, 3 species (3.2\%) treating five ailments and Albizia antunesiana used to treat six ailments (Table 1). A total of 18 medical conditions were treated using remedies made from medicinal plants (Figure 3). Gastro-intestinal disorders, sexually transmitted infections, cold, cough and sore throat and gynaecological problems were treated with the highest number of medicinal plant species (Figure 3). Gastro-intestinal disorders, particularly cholera, diarrhoea and dysentery are a major concern not only in south-central Zimbabwe but the whole country and; in Mozambique as well, where dysentery and cholera usually result in high mortality rate if not treated promptly [142]. Sexually transmitted infections are a major public health concern in developing countries with their transmission rate regarded as one of the highest in the world [143]. Sexually transmitted infections are one of the most common reasons for people to use herbal medicines and visit traditional healers in Zimbabwe $[1,4,8]$.

Plant remedies were often utilized in the form of extracts $(76.3 \%)$, sap (11.8\%) and powder (6.5\%) (Table 1). Other preparation methods included paste, chewing the raw plant and inhaling smoke or vapour generated by burning some of the medicinal plant species. Most of the preparations $(69.9 \%)$ were prescribed orally in warm water or soft porridge (Table 1). Herbal preparation methods and dosage depend on the type of disease. Some plants were boiled while others were applied directly in fresh form. Some herbal medicines were applied topically, either as bath, massage or lotion. For example, the use of burnt fruit peels of Entandrophragma caudatum which were mixed with vaseline and applied on genital areas affected with genital warts [9]. The herbal prescriptions were usually given to patients until patients reported positive results.

Monotherapy preparations made from a single plant species were the most dominant (92.5\%); $4.3 \%$ and 3.2\% of the herbal concoctions were prepared from a combination of two and three species respectively. Those that involved the use of two species included mixing roots of Annona stenophylla and Securidaca longipedunculata as snake repellent [8]. Roots of Elephantorrhiza goetzei and Piliostigma thonningii were used as remedy for bilharzia (schistosomiasis) [8]. Water extract of Eucalyptus 

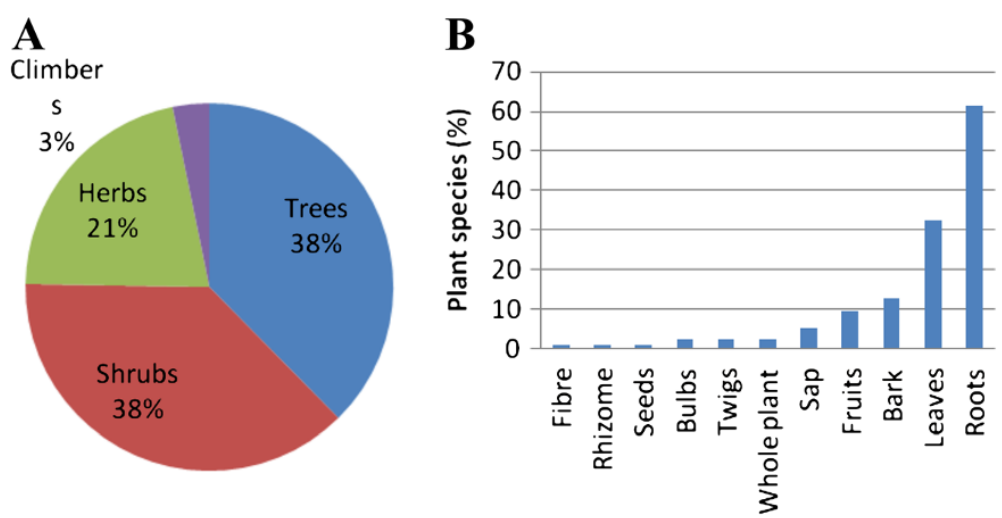

Figure $\mathbf{2}$ Characteristics of the plants used as herbal medicines in south-central Zimbabwe. (A) Growth form habit represented in pie diagram and (B) plant parts used represented in bar chart.

camaldulensis leaves, Citrus limon fruits and Psidium guajava leaves was taken as a cure for cough, flu and fever [8]. The use of multiple therapies in traditional medicine based on combining plants has recently been shown to increase the efficacy of the herbal medicine [144]. According to Bussmann and Sharon [145], the use of more than one plant species to prepare a remedy for ailments is attributed to the additive or synergistic effects that they could have during ailment treatment.

\section{Literature based proof of biological and pharmacological activities}

Out of 93 medicinal plants used in south-central Zimbabwe, 83 species (89.2\%) have proven biological and pharmacological activities (Table 1). The presence of these active ingredients in herbal medicines is directly linked to their ability to prevent or treat ailments. Analysis of the biological and pharmacological activity data (Table 1) shows the wide variety of biological activity of plants used as herbal medicines in south-central Zimbabwe; and the presence of these active ingredients in utilized plants as herbal medicines corroborates the popular traditional knowledge and medicinal uses of the documented plants. The identification of biologically active compounds in herbal medicines needs to be interpreted in the light of the traditional uses of the plants as well as herbal medicine preparation and dosage [146]. It is worth mentioning that most of these activities, mainly the antibacterial, anti-inflammatory, antiparasitic and analgesic properties; and the presence of

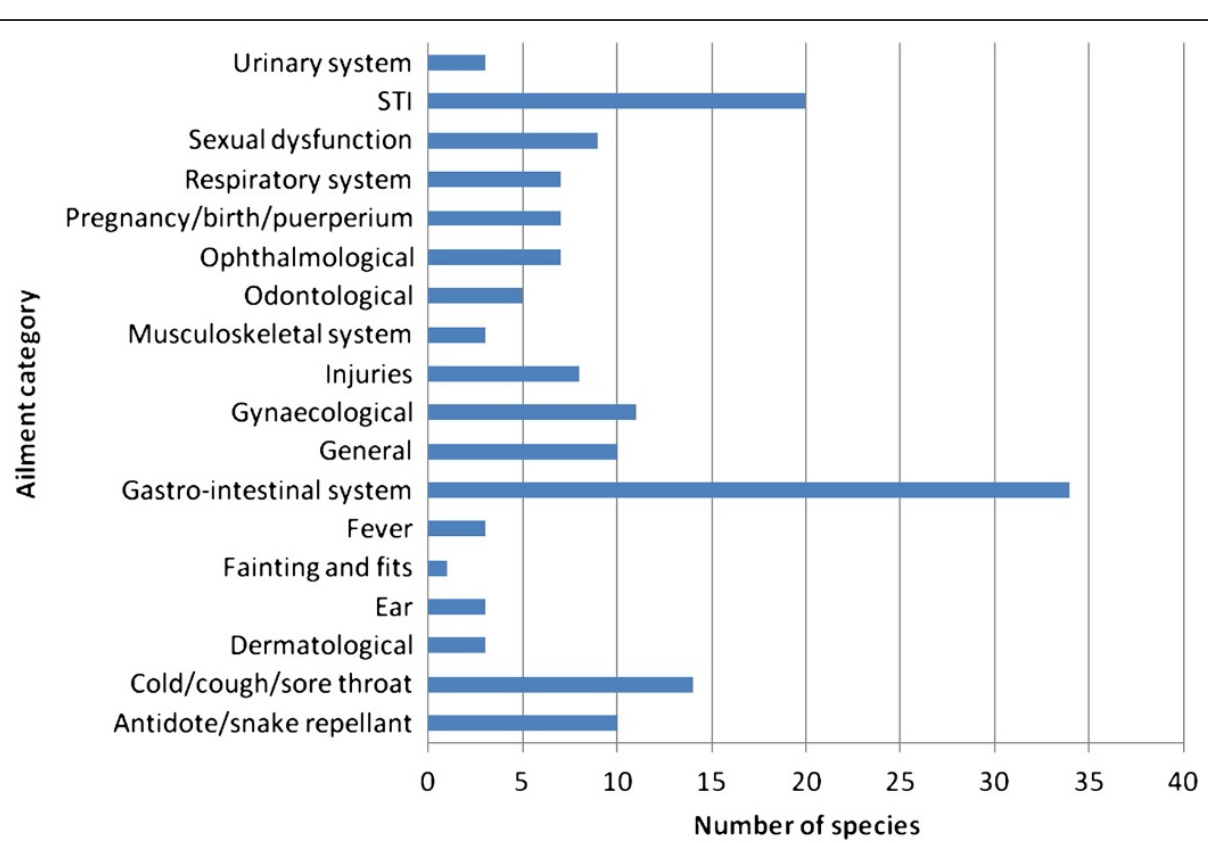

Figure 3 Major ailments and disease categories and plant species reported. Most species were reported in more than one ailment category. 
alkaloids, flavonoids, saponins and tannins confirm the different popular applications of extracts obtained from traditional medicines.

The majority (82.8\%) of the 93 plant species used as traditional medicines in south-central Zimbabwe have similar applications in other regions of Zimbabwe as well as other parts of the world (Table 1). The medicinal uses of 49 species (52.7\%) are supported by reports of similar uses elsewhere in Zimbabwe and 40 species (43\%) have similar uses in the other parts of the world (Table 1). Such similarities in the cross-cultural usage of the traditional plant remedies are a strong indication of the bioactivity potential of the documented plant species. The following 16 medicinal species (17.2\%) appear not to be popular for the ethno medicinal uses documented in Zimbabwe: Androstachys johnsonii (aphrodisiac), Brachystegia boehmii (sexually transmitted infections), Bridelia mollis (cough), Carissa bispinosa (cough and diarrhoea), Coleochloa setifera (pneumonia), Commiphora marlothii (sexually transmitted infections), Corchorus tridens (backache), Entandrophragma caudatum (genital warts), Indigofera setiflora (diarrhoea), Pellaea sp. (chest pains), Prunus persica (diarrhoea), Searsia dentata (ulcers, diarrhoea and stomach problems), Searsia pyroides (cough), Spirostachys africana (venereal infections), Strychnos madagascariensis (sore eyes) and Vitex payos (cough) (Table 1). These findings of new ethno medicinal plant uses in south-central Zimbabwe shows the importance of the documentation of such traditional indigenous knowledge. Some of these species can therefore be targeted for phytochemical and pharmacological studies with the aim of identifying active ingredients contained by such plants resulting in them having unique therapeutic uses.

This review showed substantial commonality in the general use of medicinal plants in south-central Zimbabwe, the other regions of Zimbabwe and the rest of the world. For example, ten plant species used to treat at least four ailments in south-central Zimbabwe include Albizia antunesiana (six ailments), Annona stenophylla (four ailments), Cassia abbreviata (five ailments), Elephantorrhiza goetzei (four ailments), Flueggea virosa (four ailments), Kirkia acuminata (five ailments), Peltophorum africanum (four ailments), Pterocarpus angolensis (four ailments), Sansevieria hyacinthoides (five ailments), Strychnos cocculoides (five ailments) and Ziziphus mucronata (four ailments) (Table 1). With the exception of Sansevieria hyacinthoides, the other nine species have been documented by Gelfand et al. [1] as valuable medicinal plants in most regions of Zimbabwe with at least six medicinal applications each. Literature search showed that the roots of Albizia antunesiana are widely used in tropical Africa to treat abdominal pains, cuts, depressed fontanelle in infants, gonorrhoea and other sexually transmitted diseases, infertility in women, painful and swollen legs, pneumonia, prevent abortion, sore eyes, sore throat, tonsillitis, tuberculosis and ulcers [147]. A bark infusion of Albizia antunesiana is taken to treat constipation and applied externally to cuts; whereas crushed leaves are used as an enema for their purgative action and as a dressing to treat oedema of the legs [147]. Previous research by Gelfand et al. [1] showed wide use of Annona stenophylla in traditional medicine in Zimbabwe. Infusion of Annona stenophylla root or bark is used to treat abdominal pains, boils, chest pains, constipation, diarrhoea, dysmenorrhea, hiccoughs, oedema, sexually transmitted diseases and sprains [1]. All plant parts of Cassia abbreviata are used in tropical Africa to treat gastro-intestinal disorders, bilharzia, diarrhoea, dysmenorrhea, eye problems, haematuria, headache, malaria, pneumonia, snakebites, toothache and venereal diseases [68]. Decoction of all plant parts of Cassia abbreviata are used as aphrodisiac, abortifacient, purgative, tonic and vermifuge [68]. Root infusion of Elephantorrhiza goetzei is widely used in Zimbabwe as remedy for abdominal pains, backache, bilharzia, constipation, depressed fontenelle, diarrhoea and gonorrhea [1]. Flueggea virosa is an important medicinal plant in tropical Africa, used for the treatment of a wide variety of ailments, alone or in combination with other plants. All plant parts of Flueggea virosa are used to treat frigidity, liver, bile, kidney, testicular inflammation, sterility, urinary and venereal diseases [56]. All plant parts of Kirkia acuminata are used in traditional medicine in Zimbabwe as herbal medicine for abdominal pains, antidote, cough, emetic and wounds [1]. The bark and root extracts of Peltophorum africanum are traditionally used in southern Africa to treat acute and chronic pains, boosting resistance to diseases, depression, diarrhoea, dysentery, infertility, intestinal parasites and wounds [84]. The bark of Pterocarpus angolensis is widely used in tropical Africa as an astringent to treat diarrhoea, heavy menstruation, nose bleeding, headache, stomachache, schistosomiasis, sores and skin problems [148]. Leaves, rhizomes and roots of Sansevieria hyacinthoides are widely used in tropical Africa to treat ear infections, haemorrhoids, intestinal worms, measles, prevention of miscarriage, sexually transmitted infections, stomach disorders, toothache and ulcers [31]. All plant parts of Strychnos cocculoides are widely used in Zimbabwe to treat abdominal pains, amenorrhoea, cough, diarrhoea, gonorrhea, hydrocele, infertility, sore eyes and sore throat [1]. Medicines obtained from infusion of the roots, bark, leaves and/or fruits of Ziziphus mucronata are used to treat bilharzia, boils, chronic cough, depressed fontanelle, diarrhoea, dysmenorrhoea, infertility in women, menorrhagia, oedema, pneumonia, snake bite, toothache, venereal diseases and wounds [1]. 


\section{Future research and perspectives}

This review showed that local people in south-central Zimbabwe rely on traditional medicines to treat a wide spectrum of human ailments and are knowledgeable about the identities and applications of medicinal plants. Many people in south-central Zimbabwe are still dependent on medicinal plants, at least for the treatment of basic human ailments like cold, cough, diarrhoea, fever, skin infections, sexually transmitted infections, sore eyes and tooth infections. Data collected in the present review illustrates that gastro-intestinal disorders and sexually transmitted infections are treated with the highest number of medicinal plant species. These findings correlate strongly with observations made by Ribeiro et al. [142] that cholera, diarrhoea and dysentery are a major concern in Mozambique and southern Africa as well as findings made by Van Vuuren and Naidoo [143] that sexually transmitted infections are a major public health concern in developing countries. Reports of similar medicinal applications of the documented plants in south-central Zimbabwe, other regions of Zimbabwe and the rest of the world indicate that these species are valuable sources of ethnomedicines. This comparative analysis strengthens the firm belief that traditional indigenous knowledge represent not only an important heritage, developed over the centuries, but also considerable mass of data that should be exploited in order to provide new and useful knowledge on plant resources. It is therefore, necessary to preserve this indigenous knowledge on traditional medicines by proper documentation, identification of plant species used, herbal preparation and dosage. This inventory will assist future workers on the selection of herbal plants to evaluate for phytochemical safety and pharmaceutical efficacy. There is also need for more research on the active compounds of these herbal medicines, some of which have already shown interesting biological and pharmacological activities as shown in Table 1. There is need to establish the link between the biological activity and particular compounds responsible for the wide use of these medicinal plants. The documented indigenous knowledge in south-central Zimbabwe and available scientific literature strongly suggests that at least some of the plants used as herbal medicines can be potential sources of newer drugs.

At the present moment, phytochemistry and pharmacological analysis of traditional medicines occupy a key position in medicinal plant research and indigenous knowledge systems. Sharing of such knowledge is crucial for maintaining options for the use of traditional medicines, particularly as use of alternative medicine is growing because of its moderate costs and increasing faith in herbal medicines. Significant levels of global knowledge on conventional pharmaceuticals originated from indigenous traditional knowledge. For example, many of the conventional drugs available on the market today have a long history of use as traditional medicines, among them are aspirin, opium and quinine. While south-central Zimbabwe is endowed with a strong culture of herbal medicine usage for primary health care, there is need to standardize the drug preparation, dosage and route of administration. Validating the correlations of the ethno medicinal uses, bioactive substances, biological and pharmacological effects is of special importance and is still the primary task for future research. Efforts are also needed to investigate the physiological and biochemical functions demonstrated by these species, identifying the individual bioactive natural products and illustrate their mechanisms of action.

Like most African countries, Zimbabwe is an important repository of medicinal plants usage in primary healthcare. This is reflected in the great diversity of plants used for medicinal purposes in south-central Zimbabwe as well as in the wide range of their applications and associated traditional medicine procedures. There is a growing upsurge in demand for traditional medicines in Zimbabwe for various human ailments. As demand for medicinal plants continue to accelerate, awareness creation should be made among local communities to ensure sustainable use and conservation of the medicinal plants. A collaborative approach for sustainable use, conservation and management of medicinal plants should be put into place and involve all stakeholders. Communities in south-central Zimbabwe should be actively involved in plant resource management as they depend on these natural resources for their primary healthcare needs. It is hoped that this will strike a balance between meeting their health needs and wise use of plant resources to ensure sustainable development. The most serious threats to medicinal plants, like any other forms of biodiversity are habitat loss and fragmentation, climate change and invasive species. It is not known whether over-exploitation of medicinal plants is an issue in south-central Zimbabwe. However, future studies in south-central Zimbabwe should focus on how local communities use and manage medicinal plants. Such studies will help in understanding how local communities relate to the plant resources that they use as medicines.

\section{Competing interests}

The author declares that he has no competing interests.

Authors' contributions

AM conceptualized the study and wrote the manuscript. The author read and approved the final manuscript.

\section{Acknowledgements}

The author would like to thank four anonymous reviewers for their constructive comments. 
Received: 12 February 2013 Accepted: 6 April 2013

Published: 4 May 2013

\section{References}

1. Gelfand M, Drummond RB, Mavi S, Ndemera B: The traditional medical practitioner in Zimbabwe: his principles of practice and pharmacopoeia. Gweru: Mambo Press; 1985.

2. Cunningham AB: An Investigation of the herbal medicine trade in Natal/ KwaZulu. Pietermaritzburg: Investigational Report No 29 Institute of Natural Resources; 1988

3. Hostettmann K, Marston A, Ndjoko K, Wolfender J-L: The potential of African medicinal plants as a source of drugs. Curr Organic Chem 2000, 4:973-1010.

4. Kambizi L, Afolayan AJ: An ethnobotanical study of plants used for the treatment of sexually transmitted diseases (Njovhera) in Guruve District, Zimbabwe. J Ethnopharmacol 2001, 77:5-9.

5. Mukamuri B: Use of medicinal plants in Zimbabwe's urban and rural areas. Zimbabwe Sci News 1998, 32:42-45.

6. Gerhardt K, Nemarundwe N: Participatory planting and management of indigenous trees: lessons from Chivi District, Zimbabwe. Agric Human Values 2006, 23:231-243.

7. Shoko T: Karanga traditional medicine and healing. Afr J Trad CAM 2007, 4:501-509.

8. Maroyi A: Ethnobotanical study of medicinal plants used by people in Nhema communal area, Zimbabwe. J Ethnopharmacol 2011, 136:347-354.

9. Chigora P, Masocha R, Mutenheri F: The role of indigenous medicinal knowledge (IMK) in the treatment of ailments in rural Zimbabwe: the case of Mutirikwi communal lands. J Sustainable Develop Africa 2007, 9:26-43.

10. Mabogo DEN: The ethnobotany of the VhaVenda. MSC Thesis. Pretoria: University of Pretoria; 1990.

11. Deutschländer MS, Lall N, Van De Venter M: Plant species used in the treatment of diabetes by South African traditional healers: an inventory. Pharmaceut Biol 2009, 47:348-365.

12. Van Wyk B-E, Van Oudtshoorn B, Gericke N: medicinal plants of South Africa. Pretoria: Briza Publications Pretoria; 2009.

13. Chitemerere TA, Mukanganyama S: In vitro antibacterial activity of selected medicinal plants from Zimbabwe. African J PI Sci Biotech 2011, 5:1-7.

14. Wauthoz N, Balde A, Balde ES, Van Damme M, Duez P: Ethnopharmacology of Mangifera indica L. bark and pharmacological studies of its cain C-Glucosylxanthone, Mangiferin. Int J Biomed Pharmaceut Sci 2007, 1:112-119.

15. Mølgaard P, Nielsen SB, Rasmussen DE, Drummond RB, Makaza N, Andreassen J: Anthelmintic screening of Zimbabwean plants traditionally used against Schistosomiasis. J Ethnopharmacol 2001, 74:257-264.

16. Paiva PMG, Gomes FS, Napoleão TH, Sá RA, Correia MTS, Coelho LCBB: Antimicrobial activity of secondary metabolites and lectins from plants. In Current Research, Technology and Education Topics in Applied Microbiology and Microbial Biotechnology. Edited by Mendez-Vilas A. Brazil: Formatex; 2010:396-406

17. Rea Al, Schmidt JM, Setzer WN, Sibanda S, Taylor C, Gwebu ET: Cytotoxic activity of Ozoroa insignis from Zimbabwe. Fitoterapia 2003, 74:732-735.

18. Ojewole JAO, Mawoza T, Chiwororo WDH, Owira PMO: Sclerocarya birrea (A. Rich) Hochst. [Marula] (Anacardiaceae): a review of its phytochemistry, pharmacology and toxicology and its ethnomedicinal uses. Phyto. Res 2010, 24:633-639.

19. Stafford Gl, Jäger AK, Van Staden J: African psychoactive plants. In African Natural Plant Products: New Discoveries and Challenges in Chemistry and Quality. Edited by Ho C-T, Juliani HR, Simon J. Washington, DC: ACS Symposium Series American Chemical Society; 2009:323-346.

20. Mdee LK, Yeboah SO, Abegaz BM: Rhuschalcones II-VI, five new bichalcones from the root bark of Rhus pyroides. J Nat Prod 2003, 66:599-604.

21. Dushimemaria F, Mumbengegwi DR, Du Preez I, Bock R: Qualitative phytochemical screening and in vitro antimicrobial effects of plant extracts of Searsia tenuinervis. J Res Microbes 2012, 1:88-95.

22. Munodawafa T, Chagonda LS, Viol I, Muchuweti M, Moyo SR: Total phenolic content and antioxidant activity of some Zimbabwean traditional medicinal plants. In Drug Plants 111. Edited by Govil JN, Singh VK. Houston: Studium Press LLC; 2010:363-373.

23. Patel S: Food, pharmaceutical and industrial potential of Carissa genus: an overview. Biotechnol: Rev Environ Sci; 2012
24. Mavi S: Medicinal plants and their uses in Zimbabwe. In Indigenous Knowledge and its Uses in Southern Africa. Edited by Norman H, Snyman I, Cohen M. Pretoria: Human Sciences Research Council; 1996:67-73.

25. Maroyi A: Traditional homegardens and rural livelihoods in Nhema, Zimbabwe: a sustainable agroforestry system. Int I Sust Develop World Ecol 2009, 16:1-8.

26. Schmelzer GH: Catharanthus roseus (L.) G. Don. In Plant Resources of Tropical Africa 11(1): Medicinal Plants 1. Edited by Schmelzer GH, Gurib-Fakim A. Wageningen: Prota Foundation; 2008:153-158.

27. Hassan KA, Brenda AT, Patrick V, Patrick OE: In vivo antidiarrheal activity of the ethanolic leaf extract of Catharanthus roseus Linn. (Apocyanaceae) in Wistar rats. African J Pharm and Pharmacol 2011, 5:1797-1800.

28. van de Venter M, Roux S, Bungu LC, Louw J, Crouch NR, Grace MO, Maharaj $V$, Pillay P, Sewnarian P, Bhagwandin N, Folb P: Antidiabetic screening and scoring of 11 plants traditionally used in South Africa. J Ethnopharmacol 2008, 119:81-86.

29. Msonthi JD, Magombo D: Medicinal herbs in Malaria and their uses. Hamdard Medicus 1983, 20:94-100.

30. Hassan HS, Ahmadu AA, Hassan AS: Analgesic and anti-inflammatory activities of Asparagus africanus root extract. Afr J Trad CAM 2008, $5: 27-31$

31. Takawira-Nyenya R, Stedje B: Ethnobotanical studies in the genus Sansevieria Thunb. (Asparagaceae) in Zimbabwe. Ethno Res Applicat 2011, 9:421-443.

32. Hedberg I, Staugard F: Traditional medicinal plants: traditional medicine in Botswana. Gaborone: Ipeleng Publishers; 1989.

33. Case O: An assessment of medicinal hemp plant extracts as natural antibiotic and immune modulation phytotherapies. MSc Thesis. Cape Town: University of the Western Cape; 2005.

34. Aliero AA, Jimoh FO, Afolayan AJ: Antioxidant and antibacterial properties of Sansevieria hyacinthoides. Int J Pure App/ Sci 2008, 2:103-110.

35. Botes $L$, van der Westhuizen FH, Loots DT: Phytochemical contents and antioxidant capacities of two Aloe greatheadii var. davyana extracts. Molecules 2008, 13:2169-2180.

36. Jodanus N: Brachylaena discolor DC. 2003. http://www.plantzafrica.com/ plantab/brachylaendiscol.htm.

37. Becker JV, van der Merwe MM, Van Brummelen AC, Pillay P, Crampton BG, Mmutlane EM, Parkinson C, Van Heerden FR, Crouch NR, Smith PJ, Mancama DT, Maharaj VJ: In vitro anti-plasmodial activity of Dicoma anomala subsp. gerrardii (Asteraceae): identification of its main active constituent, structure-activity relationship studies and gene expression profiling. Malar I 2011, 10:295.

38. Bussmann RW, Sharon D, Díaz DP, Barocio Y: Peruvian plants canchalagua (Schkuhria pinnata (Lam.) Kuntze), Hercampuri (Gentianella alborosea (Gilg.) Fabris) and corpus way (Gentianella bicolor (Wedd.) J. Pringle) prove to be effective in the treatment of acne. Arnaldoa 2008, 15:149-152.

39. Jimoh FO, Adedapo AA, Afolayan AJ: Comparison of the nutritive value, antioxidant and antibacterial activities of Sonchus asper and Sonchus oleraceus. Rec Nat Prod 2011, 5:29-42.

40. Paraskeva MP, Van Vuuren SF, Van Zyl RL, Davids H, Viljoen AM: The in vitro biological activity of selected South African Commiphora species. J Ethnopharmacol 2008, 119:673-679.

41. Bosch CH: Maytenus heterophylla (Eckl. \& Zeyh.) N.Robson. In Plant Resources of Tropical Africa 2: Vegetables. Edited by Grubben GJH, Denton OA. Wageningen: Prota Foundation; 2004:380-381.

42. Da Silva G, Taniça M, Rocha J, Serrano R, Gomes ET, Sepodes B: In vivo antiinflammatory effect and toxicological screening of Maytenus heterophylla and Maytenus senegalensis extracts. Hum Exp Toxicol 2000, 30:693-700.

43. Peni J, Elinge CM, Yusuf H, Itodo AU, Agaie BM, Mbongo AN, Chogo E: Phytochemical screening and antibacterial activity of Parinari curatellifolia stem extract. J Med Plant Res 2010, 4:2099-2102

44. More G, Tshikalange TE, Lall N, Botha F, Meyer JJM: Antimicrobial activity of medicinal plants against oral microorganisms. J Ethnopharmacol 2008, 119:473-477.

45. Dibwe DF, Awale S, Kadota S, Tezuka Y: Damnacanthal from the Congolese medicinal plant Garcinia huillensis has a potent preferential cytotoxicity against human pancreatic cancer PANC-1 cells. Phytother Res 2012, 26:1920-1926.

46. Magadula JJ, Suleimani HO: Cytotoxic and anti-HIV activities of some Tanzanian Garcinia species. Tanzania J Health Res 2010, 12:1-7. 
47. Wilkins-Ellert MH: Cucumis anguria L. In Plant Resources of Tropical Africa 2: Vegetables. Edited by Grubben GJH, Denton OA. Wageningen: Prota Foundation; 2004:238-242.

48. Cai L, Wei GX, van der Bijl P, Wu CD: Namibian chewing stick, Diospyros lycioides, contains antibacterial compounds against oral pathogens. J Agric Food Chem 2000, 48:909-914.

49. Mujuru L: Diospyros lycioides Desf. In Plant Resources of Tropical Africa 16: Fibres. Edited by Brink M, Achigan-Dako EG. Wageningen: Prota Foundation; 2012:126-129.

50. Adeniyi BA, Odelola HA, Oso BA: Antimicrobial potentials of Diospyros mespiliformis (Ebenaceae). Afr J Med Sci 1996, 25:221-224.

51. Pretorius JC, Magama S, Zietsman PC: Purification and identification of antibacterial compounds from Euclea crispa subsp crispa (Ebenaceae) leaves. S Afr J Bot 2003, 69:579-586.

52. Samie $A$, Obi $C L$, Bessong PO, Namrita L: Activity profiles of fourteen selected medicinal plants from rural Venda communities in South Africa against fifteen clinical bacterial species. Afr J Biotech 2005, 4:1443-1451.

53. Molotja GM, Ligavha-Mbelengwa MH, Bhat RB: Antifungal activity of root, bark, leaf and soil extracts of Androstachys johnsonii Prain. Afr J Biotech 2011, 10:5725-5727.

54. Ngueyema TA, Brusotti G, Caccialanza G, Finzi PV: The genus Bridelia: a phytochemical and ethnopharmacological review. J Ethnopharmacol 2009, 124:339-349.

55. Madureira AM, Ramalhete C, Mulhovo S, Duarte A, Ferreira M-JU: Antibacterial activity of some African medicinal plants used traditionally against infectious diseases. Pharmaceut Biol 2012, 50:481-489.

56. Tabuti JRS: Flueggea virosa (Roxb. ex Willd.) Voigt. In Plant Resources of Tropical Africa 11(1): Medicinal Plants 1. Edited by Schmelzer GH, Gurib-Fakim A. Wageningen: Prota Foundation; 2008:305-308.

57. Ramalhete C, Lopes D, Mulhovo S, Rosário VE, Ferreira MJU: Antimalarial activity of some plants traditionally used in Mozambique. In Workshop Plantas Medicinais e Fitoterapêuticas nos Trópicos. IICT/CCCM, 29, 30 e 31 de Outubro de. 2008. http://www2.iict.pt/archive/doc/ C_Ramalhete_wrkshp_plts_medic.pdf.

58. Dickson RA, Houghton PJ, Hylands PJ, Gibbons S: Antimicrobial, resistancemodifying effects, antioxidant and free radical scavenging activities of Mezoneuron benthamianum Baill., Securinega virosa Roxb. \& Willd. and Microglossa pyrifolia Lam. Phytoth Res 2006, 20:41-45.

59. Moshi MJ, Kapingu MC, Uiso FC, Mbwambo ZH, Mahunnah RLA: Some pharmacological properties of an aqueous extract of Securinega virosa roots. Pharmaceut Biol 2000, 38:214-221.

60. Ragunathan M, Solomon M: The study of spiritual remedies in orthodox rural churches and traditional medicinal practice in Gondar Zuria district, Northwestern Ethiopia. 2009. http://www.emanuscript.in/sample_1.pdf.

61. Mmushi TJ: Screening, isolation and purification of bioactive compounds with antibacterial activity against Mycobacterium smegmatis. MSC Thesis. Mangweng: University of Limpopo; 2011.

62. Ilavarasan J, Mallika M, Venkataraman S: Anti-inflammatory and free radical scavenging activity of Ricinus communis root extract. J Ethnopharmacol 2006, 103:478-480.

63. Jena J, Gupta AK: Ricinus communis Linn: a phytopharmacological review. Int J Pharm Pharmaceut Sci 2012, 4:25-29.

64. Mulaudzi RB, Ndhlala AR, Kulkarni MG, Finnie JF, Van Staden J: Antimicrobial properties and phenolic contents of medicinal plants used by the Venda people for conditions related to venereal diseases. J Ethnopharmacol 2011, 135:330-337.

65. Amoo SO, Aremu AO, Moyo M, Van Staden J: Antioxidant and acetylcholinesterase-inhibitory properties of long-term stored medicinal plants. BMC Complement Altern Med 2012, 12:87.

66. Adedapo AA, Sofidiya MO, Masika PJ, Afolayan AJ: Anti-inflammatory and analgesic activities of the aqueous extract of Acacia karroo stem bark in experimental animals. Basic Clin Pharmacol Toxicol 2008, 103:397-400.

67. Nielsen TRH, Kuete V, Jäger AK, Meyer JJM, Lall N: Antimicrobial activity of selected South African medicinal plants. BMC Compl Altern Med 2012, $12: 74$

68. Kawanga V: Cassia abbreviata Oliv. In Plant Resources of Tropical Africa 11(1): Medicinal Plants 1. Edited by Schmelzer GH, Gurib-Fakim A. Wageningen: Prota Foundation; 2008:144-146.

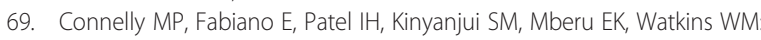
Antimalarial activity in crude extracts of Malawian medicinal plants. Ann Trop Med Parasitol 1996, 90:597-602.
70. Leteane MM, Ngwenya BN, Muzila M, Namushe A, Mwinga J, Musonda R, Moyo S, Mengestu YB, Abegaz BM, Andrae-Marobela K: Old plants newly discovered: Cassia sieberiana D.C. and Cassia abbreviata Oliv. Oliv.root extracts inhibit in vitro HIV-1c replication in peripheral blood mononuclear cells (PBMCs) by different modes of action. J Ethnopharmacol 2012, 141:48-56.

71. Lemmens RHMJ: Dalbergia melanoxylon Guill. \& Perr. In Plant Resources of Tropical Africa 7(1): Timbers 1. Edited by Louppe D, Oteng-Amoako AA, Brink M. Wageningen: Prota Foundation; 2008:202-207.

72. Gundidza M, Gaza N: Antimicrobial activity of Dalbergia melanoxylon extracts. J Ethnopharmacol 1993, 40:127-130.

73. Wanjala CC, Majinda RR: A new stilbene glycoside from Elephantorrhiza goetzei. Fitoterapia 2001, 72:649-655.

74. Omer MEA, Al Magboul AZ, El Egami AA: Sudanese plants used in folkloric medicine: screening for antibacterial activity. Part IX. Fitoterapia 1998, 69:542-545.

75. Taniguchi M, Kubo I: Ethnobotanical drug discovery based on medicine men's trials in the african savanna: screening of east African plants for antimicrobial activity II. J Nat Prod 1993, 56:1539-1546.

76. Nguyen PH, Le TV, Thuong PT, Dao TT, Ndinteh DT, Mbafor JT, Kang KW, Oh WK: Cytotoxic and PTP1B inhibitory activities from Erythrina abyssinica. Bioorg Med Chem Lett 2009, 19:6745-6749.

77. Okeleye BI, Samie A, Bessong PO, Mkwetshana NF, Green E, Clarke AM, Ndip RN: Crude ethyl acetate extract of the stem bark of Peltophorum africanum (Sond, Fabaceae) possessing in vitro inhibitory and bactericidal activity against clinical isolates of Helicobacter pylori. J Med Plant Res 2010, 4:1432-1440.

78. Ighodaro OM, Agunbiade SO, Omole JO, Kuti OA: Evaluation of the chemical, nutritional, antimicrobial and antioxidant-vitamin profiles of Piliostigma thonningii leaves (Nigerian species). Res J Med Plants 2012, 6:537-543.

79. Samie A, Housein A, Lall N, Meyer JJ: Crude extracts of, and purified compounds from, Pterocarpus angolensis, and the essential oil of Lippia javanica: their in-vitro cytotoxicities and activities against selected bacteria and Entamoeba histolytica. Ann Trop Med Parasitol 2009, 103:427-439.

80. Kota GC, Karthikeyan M, Kannan M, Rajasekar: Flacourtia indica (Burm. f.) Merr.: a phytopharmacological review. Int J Res Pharmaceut Biomed Sci 2012, 3:78-81.

81. Marini-Bettolo GB, Patamia M, Nicoletti M, Galeffi C, Messana I: Research on African medicinal plants ii. Hypoxosie, a new glucoside of uncommon structure from Hypoxis obtusa Burch. Tetrahedron 1982, 38:1683-1687.

82. Msonthi JD, Toyota M, Marston A, Hostettmann K: A phenolic glycoside from Hypoxis obtusa. Phytochem 1990, 29:3977-3979.

83. Akah PA, Odo Cl: Hepatoprotective effect of the solvent fractions of the stem of Hoslundia opposita Vahl (Lamiaceae) against carbon tetrachloride- and paracetamol-induced liver damage in rats. Int J Green Pharm 2010, 4:54-58.

84. Watt JM, Breyer-Brandwijk MG: The medicinal and poisonous plants of southern and eastern Africa. London: E \& S Livingstone; 1962.

85. Gundidza GM, Deans SG, Svoboda KP, Mavi S: Antimicrobial activity of essential oil from Hoslundia opposita. Cent Afr J Med 1992, 38:290-293.

86. El-Ansari MA, Aboutabl EA, Farrag ARH, Sharaf M, Hawas UW, Soliman GM, El-Seed GS: Phytochemical and pharmacological studies on Leonotis leonurus. Pharm Biol 2009, 47:894-902.

87. Nyamoita MG: Anti-larval compounds from Vitex schiliebenii and Vitex payos. MSc Thesis. Nairobi: Kenyatta University; 2011.

88. Batista R, Júnior AS, De Oliveira AB: Plant-derived antimalarial agents: new leads and efficient phytomedicines. Part II. non-alkaloidal natural products. Molecules 2009, 14:3037-3072.

89. Hoet S, Pieters L, Muccioli GG, Habib-Jiwan JL, Opperdoes FR, QuetinLeclercq J: Antitrypanosomal activity of triterpenoids and sterols from the leaves of Strychnos spinosa and related compounds. J Nat Products 2007, 70:1360-1363.

90. Orwa C, Mutua A, Kindt R, Jamnadass R, Anthony S: Agroforestree database: a tree reference and selection guide version 4.0. 2009. http://www.worldagroforestrycentre. org/sea/products/afdbases/af/asp/SpeciesInfo.asp?SplD=272.

91. Chávez D, Chai H-B, Chagwedera TE, Gao Q, Farnsworth NR, Cordell GA, Pezzuto JM, Kinghorn AD: Novel stilbenes isolated from the root bark of Ekebergia benguelensis. Tetrahedron Lett 2001, 42:3685-3688.

92. Jonker SA, Nkunya MHH, Mwamtobe L, Geenevase J, Koomen G-J: A new coumarin and polyhydroxysqualenes from Ekebergia benguelensis. Nat Prod Lett 1997, 10:245-248. 
93. Ansell SM, Taylor DAH: Limonoids from the seed of Entandrophragma caudatum. Phytochem 1988, 27:1218-1220.

94. Grosvenor PW, Gothard PK, McWilliams NC, Supriono A, Gray DO: Medicinal plants from Riau Province, Sumatra, Indonesia, part i: uses. J Ethnopharmacol 1995, 45:75-95.

95. Aiyelero OM, Ibrahim ZG, Yaro AH: Analgesic and anti-inflammatory properties of the methanol leaf extract of Ficus ingens (Moraceae) in rodents. Nigerian J Pharm Sci 2009, 8:79-86.

96. Eldeen IMS, Elgorashi EE, Van Staden J: Antibacterial, anti-inflammatory, anti-cholinesterase and mutagenic effects of extracts obtained from some trees used in South African traditional medicine. J Ethnopharmacol 2005, 102:457-464.

97. Lumbile AU, Mogotsi KK: Ficus sur Forssk. In Plant Resources of Tropical Africa 7(1): Timbers 1. Edited by Louppe D, Oteng-Amoako AA, Brink M. Wageningen: Prota Foundation; 2008:285-288.

98. Olusesan AG, Ebele OC-L, Onwuegbuchulam ON, Olorunmola EJ: Preliminary in-vitro antibacterial activities of ethanolic extracts of Ficus sycomorus Linn. and Ficus platyphylla Del. (Moraceae). Afr J Microbiol Res 2010, 4:598-601.

99. Sandabe UK, Onyeyili PA, Chibuzo GA: Phytochemical screening and effect of aqueous extract of Ficus sycomorus L. (Moraceae) stembark on muscular activity in laboratory animals. J Ethnopharmacol 2006, 103:481-483.

100. Monera TG, Maponga CC: Prevalence and patterns of Moringa oleifera use among HIV positive patients in Zimbabwe: a cross-sectional survey. J Public Health Afr 2012, 3:22-24.

101. Goyal BR, Agrawal BB, Goyal RK, Mehta AA: Phyto-pharmacology of Moringa oleifera Lam.: an overview. Natural Prod Radiance 2007, 6:347-353.

102. Molefe-Khamanga DM, Mooketsi NA, Matsabisa MG, Kensley RM: Qualitative phytochemical studies of solvent extracts from Myrothamnus flabellifolius. Online Int J Med PI Res 2012, 1:1-5.

103. Motlhanka DMT, Mathapa G: Antioxidant activities of crude extracts from medicinal plants used by diabetic patients in Eastern Botswana. J Med Plant Res 2012, 6:5460-5463.

104. Van Wyk B-E, Viljoen AM, Klepser ME, Ernst EJ, Keele D, Roling E, Van Vuuren S, Demirci B, Baúer KHC: The composition and antimicrobial activity of the essential oil of the resurrection plant Myrothamnus flabellifolius. S Afr J Bot 2002, 68:100-105.

105. Bruneton J: Pharmacognosy: phytochemistry, medicinal plants. London: Intercept Ltd; 1999.

106. Topco G, Yapar G, Turkmen Z, Goren GC, Oksuz S, Schilling JK, Kingston DGI: Ovarian antiproliferative activity directed isolation of triterpenoids from fruits of Eucalyptus camaldulensis Dehnh. Phytochem Lett 2011, 4:421-425.

107. Babayi H, Kolo I, Okogun JI, ljah UJJ: The antimicrobial activities of methanolic extracts of Eucalyptus camaldulensis and Terminalia catappa against some pathogenic microorganisms. Biokemistri 2004, 16:106-111.

108. Gutiérrez RMP, Mitchell S, Solis RV: Psidium guajava: a review of its traditional uses, phytochemistry and pharmacology. J Ethnopharmacol 2008, 117:1-27.

109. Lozoya X, Bercerril G, Martinez M: Intraluminal perfusion model of in vitro guinea pig ileum as a model of study of the antidiarrheic properties of the guava (Psidium guajava). Arch Invest Med (Mex) 1990, 21:155-162.

110. Jaiarj P, Khoohaswan P, Wongkrajang Y, Peungvicha P, Suriyawong P, Saraya MLS, Ruangsomboon O: Anticough and antimicrobial activities of Psidium guajava Linn. leaf extract. J Ethnopharmacol 1999, 67:203-212.

111. Jaiarj $P$, Wongkrajang $Y$, Thongpraditchote $S$, Peungvicha $P$, Bunyapraphatsara N, Opartkiattikul N: Guava leaf extract and topical haemostasis. Phytother Res 2000, 14:388-391.

112. Qian H, Nihorimbere V: Antioxidant power of phytochemicals from Psidium guajava leaf. J Zhejiang Univ Sci 2004, 5:676-683.

113. Makhafola TJ, Eloff JN: Five Ochna species have high antibacterial activity and more than ten antibacterial compounds. S Afr J Sci 2012, 108. Art. \#689, http://dx.doi.org/10.4102/sajs.v108i1/2.689.

114. Maikai VA, Kobo PI, Maikai BVO: Antioxidant properties of Ximenia americana. Afr J Biotech 2010, 9:7744-7746.

115. Mulaudzi RB, Ndhlala AR, Kulkarni MG, Van Staden J: Pharmacological properties and protein binding capacity of phenolic extracts of some Venda medicinal plants used against cough and fever. J Ethnopharmacol 2012, 143:185-193

116. Marx J: Investigation of the cytotoxic potential of aqueous extracts of Dicerocaryum zanguebarium and Urginea sanguinea in vitro. MSc Thesis. Pretoria: University of Pretoria; 2005.
117. Ojewole JA: Analgesic, anti-inflammatory and hypoglycaemic effects of Securidaca longepedunculata (Fresen.) [Polygalaceae] root-bark aqueous extract. Inflammopharmacol 2008, 16:174-181.

118. Gundidza M, Sibanda M: Antimicrobial activities of Ziziphus abyssinica and Berchemia discolor. Cent Afr J Med 1991, 37:80-83.

119. Chin YW, Mdee LK, Mbwambo ZH, Mi Q, Chai HB, Cragg GM, Swanson SM, Kinghorn AD: Prenylated flavonoids from the root bark of Berchemia discolor, a Tanzanian medicinal plant. J Nat Prod 2006, 69:1649-1652.

120. Olajuyigbe OO, Afolayan AJ: Antimicrobial potency of the ethanolic crude bark extract of Ziziphus mucronata Willd. subsp. mucronata Willd. Afr J Pharm Pharmacol 2012, 6:724-730.

121. Christabel PH, Nishaa S, Vishnupriya M, Sasikumar JM, Gopalakrishnan VK: In vitro antioxidant studies and the scavenging potential of pulp and peel of Prunus persica i. fruit on different solvent systems. World J Pharm Res 2012, 1:1371-1386.

122. Kim Y, Koo B, Gong D, Lee Y, Ko J, Kim C: Comparative effect of Prunus persica L. BATSCH-water extract and tacrine (9-amino-1,2,3,4tetrahydroacridine hydrochloride) on concentration of extracellular acetylcholine in the rat hippo-campus. J Ethnopharmacol 2003, 87:149-154.

123. Orwa C, Mutua A, Kindt R, Jamnadass R, Simons A: Agroforestree database: a tree reference and selection guide version 4.0. 2009. http://www. worldagroforestrycentre.org/sea/products/afdbases/af/asp/SpeciesInfo.asp? SplD $=17989$

124. Halilu ME, Abubakar A, Garba MK, Isah AA: Antimicrobial and preliminary phytochemical studies of methanol extract of root bark of Crossopteryx febrifuga (Rubiaceae). J Appl Pharm Sci 2012, 2:66-70.

125. Salawu OA, Chindo BA, Tijani AY, Adzu B: Analgesic, anti-inflammatory, anti-pyretic and antiplasmodial effects of the methanolic extract of Crossopteryx febrifuga. J Med Plant Res 2008, 2:213-218.

126. De Boer HJ, Kool A, Broberg A, Mziray WR, Hedberg I, Levenfors JJ: Antifungal and anti-bacterial activity of some herbal remedies from Tanzania. J Ethnopharmacol 2005, 96:461-469.

127. Mbukwa E, Chacha M, Majinda RRT: Phytochemical constituents of Vangueria infausta: their radical scavenging and antimicrobial activities. Arkivoc 2007, 9:104-112.

128. Arias BÁ, Ramón-Laca L: Pharmacological properties of citrus and their ancient and medieval uses in the Mediterranean region. J Ethnopharmacol 2005, 97:89-95.

129. Chen YK, Li XS, Yang GY, Chen ZY, Hu QF, Miao MM: Phenolic compounds from Nicotiana tabacum and their biological activities. J Asian Nat Prod Res 2012, 14:450-456.

130. Shvets SA, Gutsu ON, Kintia PK: Steroidal glycosides from Nicotiana tabacum L. seeds and their biological activity. Adv Exp Med Biol 1996, 405:247-257.

131. Matu EN: Solanum incanum L. In Plant Resources of Tropical Africa 11(1): Medicinal Plants 1. Edited by Schmelzer GH, Gurib-Fakim A. Wageningen: Prota Foundation; 2008:525-528.

132. Manase MJ, Mitaine-Offer AC, Pertuit D, Miyamoto T, Tanaka C, Delemasure S, Dutartre P, Duchamp O, Mirjolet JF, Lacaille-Dubois MA: Solanum incanum and $\mathrm{S}$. heteracanthum as sources of biologically active steroid glycosides: confirmation of their synonymy. Fitoterapia 2012, 83:1115-1119.

133. Kapoor BBS, Acharya S, Mishra R: Antimicrobial screening of some medicinal plants of the Rajasthan desert. In 3rd International Conference on Biology, Environment and Chemistry IPCBEE Volume 46. Singapore: IACSIT Press. 2012. http://www.ipcbee.com/vol46/023-ICBEC2012-G20009.pdf.

134. Jaspers WJMM, Bashir AK, Zwaving JH, Malingré TM: Investigation of Grewia bicolor Juss. J Ethnopharmacol 1986, 17:205-211.

135. Ghisalberti EL: Lantana camara L. (Verbenaceae). Fitoterapia 2000, 71:467-486.

136. Manenzhe NJ, Potgieter N, Van Ree T: Composition and antimicrobial activities of volatile components of Lippia javanica. Phytochemistry 2004, 65:2333-2336

137. Burkill HM: The useful plants of West Tropical Africa. Kew: Royal Botanic Gardens; 1995

138. Koné WM, Atindehou KK, Terreaux C, Hostettmann K, Traoré D, Dosso M: Traditional medicine in North Côte d'Ivoire: screening of 50 medicinal plants for antibacterial activity. J Ethnopharmacol 2004, 93:43-49.

139. Malhotra S, Singh AP: Medicinal properties of Ginger (Zingiber officinale Rosc.). Natural Product Radiance 2003, 2:296-301.

140. Ali BH, Blunden G, Tanira MO, Nemmar A: Some phytochemical, pharmacological and toxicological properties of ginger (Zingiber officinale Roscoe): a review of recent research. Food Chem Toxicol 2008, 46:409-420 
141. Flatie T, Gedif T, Asres K, Gebre-Mariam T: Ethnomedicinal survey of Berta ethnic group Assosa Zone, Benishangul-Gumuz regional state, mid-west Ethiopia. J Ethnobiol Ethnomed 2009, 5:14.

142. Ribeiro A, Romeiras MM, Tavares J, Faria MT: Ethnobotanical survey in Canhane village, district of Massingir, Mozambique: medicinal plants and traditional knowledge. J Ethnobiol Ethnomed 2010, 6:33.

143. Van Vuuren SF, Naidoo D: An antimicrobial investigation of plants used traditionally in southern Africa to treat sexually transmitted infections. J Ethnopharmacol 2010, 130:552-558.

144. Zonyane S, Van Vuuren SF, Makunga NP: Pharmacological and phyto-chemical analysis of a medicinal plant mixture that is used as traditional medicine in Western Cape. In Paper presented at South Africa Association of Botanist 38th Annual Conference, 15-18 January 2012. Pretoria: University of Pretoria; 2012:124.

145. Bussmann RW, Sharon D: Traditional medicinal plant use in Northern Peru: tracking two thousand years of healing culture. J Ethnobiol Ethnomed 2006, 2:47.

146. Holmstedt BR, Bruhn JG: Ethnopharmacology: a challenge. In Ethnobotany, Evolution of a Discipline. Edited by Schultes RE, Von Reis S. Portland: Dioscorides Press; 1995:338-343.

147. Lemmens RHMJ: Albizia antunesiana Harms. In Plant Resources of Tropical Africa 7(1): Timbers 1. Edited by Louppe D, Oteng-Amoako AA, Brink M. Wageningen: Prota Foundation; 2007:45-46.

148. Takawira-Nyenya R: Pterocarpus angolensis DC. In Plant Resources of Tropical Africa 3: Dyes and Tannins. Edited by Jansen PCM, Cardon D. Wageningen: Prota Foundation; 2005:126-130.

\section{Submit your next manuscript to BioMed Central and take full advantage of:}

- Convenient online submission

- Thorough peer review

- No space constraints or color figure charges

- Immediate publication on acceptance

- Inclusion in PubMed, CAS, Scopus and Google Scholar

- Research which is freely available for redistribution 